\title{
Genome-wide parent-of-origin DNA methylation analysis reveals the intricacies of human imprinting and suggests a germline methylation-independent mechanism of establishment
}

\author{
Franck Court, ${ }^{1,15}$ Chiharu Tayama, ${ }^{2,15}$ Valeria Romanelli, ${ }^{1,15}$ Alex Martin-Trujillo, ${ }^{1,15}$ \\ Isabel Iglesias-Platas, ${ }^{3}$ Kohji Okamura, ${ }^{4}$ Naoko Sugahara, ${ }^{2}$ Carlos Simón, ${ }^{5}$ Harry Moore, ${ }^{6}$ \\ Julie V. Harness, ${ }^{7}$ Hans Keirstead, ${ }^{7}$ Jose Vicente Sanchez-Mut, ${ }^{8}$ Eisuke Kaneki, ${ }^{9}$ \\ Pablo Lapunzina, ${ }^{10}$ Hidenobu Soejima, ${ }^{11}$ Norio Wake, ${ }^{9}$ Manel Esteller, ${ }^{8,12,13}$ \\ Tsutomu Ogata, ${ }^{14}$ Kenichiro Hata, ${ }^{2}$ Kazuhiko Nakabayashi, ${ }^{2,16,17}$ and David Monk ${ }^{1,16,17}$ \\ ${ }^{1-14}$ [Author affiliations appear at the end of the paper.]
}

\begin{abstract}
Differential methylation between the two alleles of a gene has been observed in imprinted regions, where the methylation of one allele occurs on a parent-of-origin basis, the inactive X-chromosome in females, and at those loci whose methylation is driven by genetic variants. We have extensively characterized imprinted methylation in a substantial range of normal human tissues, reciprocal genome-wide uniparental disomies, and hydatidiform moles, using a combination of wholegenome bisulfite sequencing and high-density methylation microarrays. This approach allowed us to define methylation profiles at known imprinted domains at base-pair resolution, as well as to identify 21 novel loci harboring parent-of-origin methylation, 15 of which are restricted to the placenta. We observe that the extent of imprinted differentially methylated regions (DMRs) is extremely similar between tissues, with the exception of the placenta. This extra-embryonic tissue often adopts a different methylation profile compared to somatic tissues. Further, we profiled all imprinted DMRs in sperm and embryonic stem cells derived from parthenogenetically activated oocytes, individual blastomeres, and blastocysts, in order to identify primary DMRs and reveal the extent of reprogramming during preimplantation development. Intriguingly, we find that in contrast to ubiquitous imprints, the majority of placenta-specific imprinted DMRs are unmethylated in sperm and all human embryonic stem cells. Therefore, placental-specific imprinting provides evidence for an inheritable epigenetic state that is independent of DNA methylation and the existence of a novel imprinting mechanism at these loci.
\end{abstract}

[Supplemental material is available for this article.]

Genomic imprinting is a form of epigenetic regulation that results in the expression of either the maternally or paternally inherited allele of a subset of genes (Ramowitz and Bartolomei 2011). This imprinted expression of transcripts is crucial for normal mammalian development. In humans, loss-of-imprinting of specific loci results in a number of diseases exemplified by the reciprocal growth phenotypes of the Beckwith-Wiedemann and Silver-Russell syndromes, and the behavioral disorders Angelman and Prader-Willi syndromes (Kagami et al. 2008; Buiting 2010; Choufani et al. 2010; Eggermann 2010; Kelsey 2010; Mackay and Temple 2010). In addition, aberrant imprinting also contributes to multigenic disorders associated with various complex traits and cancer (Kong et al. 2009; Monk 2010).

Imprinted loci contain differentially methylated regions (DMRs) where cytosine methylation marks one of the parental

\footnotetext{
${ }^{15}$ These authors contributed equally to this work.

16 These authors jointly directed this work.

${ }^{17}$ Corresponding authors

E-mail nakabaya-k@ncchd.go.jp

E-mail dmonk@idibell.cat

Article published online before print. Article, supplemental material, and publication date are at http://www.genome.org/cgi/doi/10.1101/gr.164913.113.
}

alleles, providing cis-acting regulatory elements that influence the allelic expression of surrounding genes. Some DMRs acquire their allelic methylation during gametogenesis, when the two parental genomes are separated, resulting from the cooperation of the de novo methyltransferase DNMT3A and its cofactor DNMT3L (Bourc'his et al. 2001; Hata et al. 2002). These primary, or germline imprinted DMRs are stably maintained throughout somatic development, surviving the epigenetic reprogramming at the oocyteto-embryo transition (Smallwood et al. 2011; Smith et al. 2012). To confirm that an imprinted DMR functions as an imprinting control region (ICR), disruption of the imprinted expression upon genetic deletion of that DMR, either through experimental targeting in mouse or that which occurs spontaneously in humans, is required. A subset of DMRs, known as secondary DMRs, acquire methylation during development and are regulated by nearby germline DMRs in a hierarchical fashion (Coombes et al. 2003; Lopes et al. 2003; Kagami et al. 2010).

\footnotetext{
(c) 2014 Court et al. This article is distributed exclusively by Cold Spring Harbor Laboratory Press for the first six months after the full-issue publication date (see http://genome.cshlp.org/site/misc/terms.xhtml). After six months, it is available under a Creative Commons License (Attribution-NonCommercial 3.0 Unported), as described at http://creativecommons.org/licenses/by-nc/3.0/.
} 
With the advent of large-scale, base-resolution methylation technologies, it is now possible to discriminate allelic methylation dictated by sequence variants from imprinted methylation. Yet our knowledge of the total number of imprinted DMRs in humans, and their developmental dynamics, remains incomplete, hampered by genetic heterogeneity of human samples.

Here we present high-resolution mapping of human imprinted methylation. We performed whole-genome-wide bisulfite sequencing (WGBS) on leukocyte-, brain-, liver-, and placenta-derived DNA samples to identify partially methylated regions common to all tissues consistent with imprinted DMRs. We subsequently confirmed the partial methylated states in tissues using high-density methylation microarrays. The parental origin of methylation was determined by comparing microarray data for DNA samples from reciprocal genome-wide uniparental disomy (UPD) samples, in which all chromosomes are inherited from one parent (Lapunzina and Monk 2011), and androgenetic hydatidiform moles, which are created by the fertilization of an oocyte lacking a nucleus by a sperm that endoreduplicates. The use of uniparental disomies and hydatidiform moles meant that our analyses were not subjected to genotype influences, enabling us to characterize all known imprinted DMRs at base-pair resolution and to identify 21 imprinted domains, which we show are absent in mice. Lastly, we extended our analyses to determine the methylation profiles of all imprinted DMRs in sperm, stem cells derived from parthenogenetically activated metaphase-2 oocyte blastocytes (phES) (Mai et al. 2007; Harness et al. 2011), and stem cells (hES) generated from both six-cell blastomeres and the inner cell mass of blastocysts, delineating the extent of embryonic reprogramming that occurs at these loci during human development.

\section{Results}

\section{Characterization of parent-of-origin methylation profiles in human tissues using high-resolution approaches}

We combined whole-genome bisulfite sequencing with Illumina Infinium HumanMethylation 450K BeadChip arrays to generate methylation profiles. To validate this approach, we compared the DNA methylation profiles generated by each method. Methylation scores produced by the two methods are very similar when the same DNA samples were assessed by both techniques (linear regression WGBS vs. Infinium array: leukocytes $\mathrm{R}^{2}=0.92$; brain $\mathrm{R}^{2}=$ 0.91; placenta $\mathrm{R}^{2}=0.92$ ) (Supplemental Fig. S1). To determine the similarity between normal biparental leukocytes and those from reciprocal genome-wide UPDs, we compared the methylation values obtained from the Infinium array. This revealed high correlations between samples, indicating that the DNAs were similar, differing only at imprinted loci (linear regression: leukocytes vs. leukocytes $\mathrm{R}^{2}=0.95-0.98$; mean control leukocytes vs. mean pUPD $\mathrm{R}^{2}=0.98$; mean control leukocytes vs. $\mathrm{mUPD}^{2}=0.98$; mUPD vs. mean pUPD $R^{2}=0.97$; F-statistics $P<0.001$ ).

Before we attempted to discover novel imprinted DMRs in the human genome, we wished to determine the effectiveness of the Infinium array to identify known imprinted DMRs. Loci were identified which contained at least three Infinium probes with an average minimal difference of $0.3 \beta$-values (absolute methylation difference $>30 \%$ ) between reciprocal genome-wide UPD leukocyte samples, and with a prerequisite that the $\beta$-values for normal leukocytes should be between these extremes. Using these criteria, we identified 818 windows that could be merged into 145 regions harboring 576 probes incorporating 30 known DMRs within 25 imprinted domains (Table 1; Fig. 1A) (Limma linear model $P<$ $0.05)$, and presented an intermediate methylation profile in all somatic tissues (Fig. 1B). The only imprinted DMRs not found using this approach were the IG-DMR located between MEG3 and DLK1 on chromosome 14, as this region does not have probes on this array platform and IGF2-DMR0 only contains a single probe.

\section{Identification of new DMRs within known imprinted domains}

In addition to the known imprinted DMRs, the Infinium array screen of reciprocal UPDs and tissues samples uncovered several previously unidentified DMRs located within existing imprinted domains. We discovered four maternally methylated CpG islands located between the SNRPN and NDN genes on chromosome 15, a region associated with the Angelman and Prader-Willi syndromes. The methylation profiles at the SNRPN, NDN, and MAGEL2 promoters are well-established (El-Maarri et al. 2001; Sharp et al. 2010). However, little is known about the intervening $\sim 1-\mathrm{Mb}$ gene-poor region, which is likely to have arisen from an ancient duplication event, since these novel DMRs share $97.8 \%$ sequence identity with additional CpG-rich regions in the interval. We confirm the maternal methylation at these four regions using bisulfite PCR and sequencing, incorporating heterozygous SNPs in brain and leukocyte DNA (Supplemental Fig. S2A). Further analysis of this region revealed that the promoter region for MKRN3 and MIR4508 are also differentially methylated.

Extending our analysis to imprinted domains on other autosomes, we identified an $\sim 600$-bp interval of maternal methylation $4 \mathrm{~kb} \mathrm{3'}$ from the ZNF597 gene (Fig. 1C). Although the promoter of ZNF597 is a paternally methylated bidirectional silencer presumably responsible for regulating the imprinted expression of both ZNF597 and NAA60 (previously known as NAT15), this region is unlikely to be the ICR for the domain as its methylation is somatically acquired (Nakabayashi et al. 2011). In addition, WGBS and Infinium array data sets revealed a maternally methylated DMR within intron 2 of MEG8 within the chromosome 14 imprinted domain (Supplemental Fig. S2B). Lastly, we identify two maternally methylated regions. The first is an $\sim 1-\mathrm{kb}$ CpG island overlapping the promoter of isoform 3 of the ZNF331 gene, and the second coincides with exon 2 of DIRAS3 (Supplemental Fig. S2C).

\section{Genome-wide methylation profiling identifies novel imprinted domains}

To determine if there are additional imprinted DMRs in the human genome, we screened for regions of intermediate methylation common to lymphocyte, brain, and liver WGBS data sets. Using a sliding window approach that takes into account 25 consecutive CpG sites and following removal of class 1 transposable elements (LINEs, Alu/SINEs, and LTR elements) and satellite DNA, we identified 356 nonoverlapping, single-copy regions in pairwise comparisons of tissues, of which 63 loci were common to the all tissues $(0.25<$ mean $\pm 1.5 \mathrm{SD}<0.75)$ (Fig. 2A; Supplemental Table S1).

A screen for three consecutive partially methylated probes in leukocyte, brain, liver, kidney, and muscle Infinium data sets, with a profile consistent with parent-of-origin methylation in the reciprocal UPD leukocyte samples, identified 116 regions (Supplemental Table S1). By combining the 356 regions detected by WGBS and the 116 loci identified by the Infinium array, we identified 64 regions in common, which included all known imprinted DMRs and $17 \mathrm{CpG}$-rich sequences possessing a methylation profile consistent with imprinting. Using standard bisulfite PCR, we assessed 


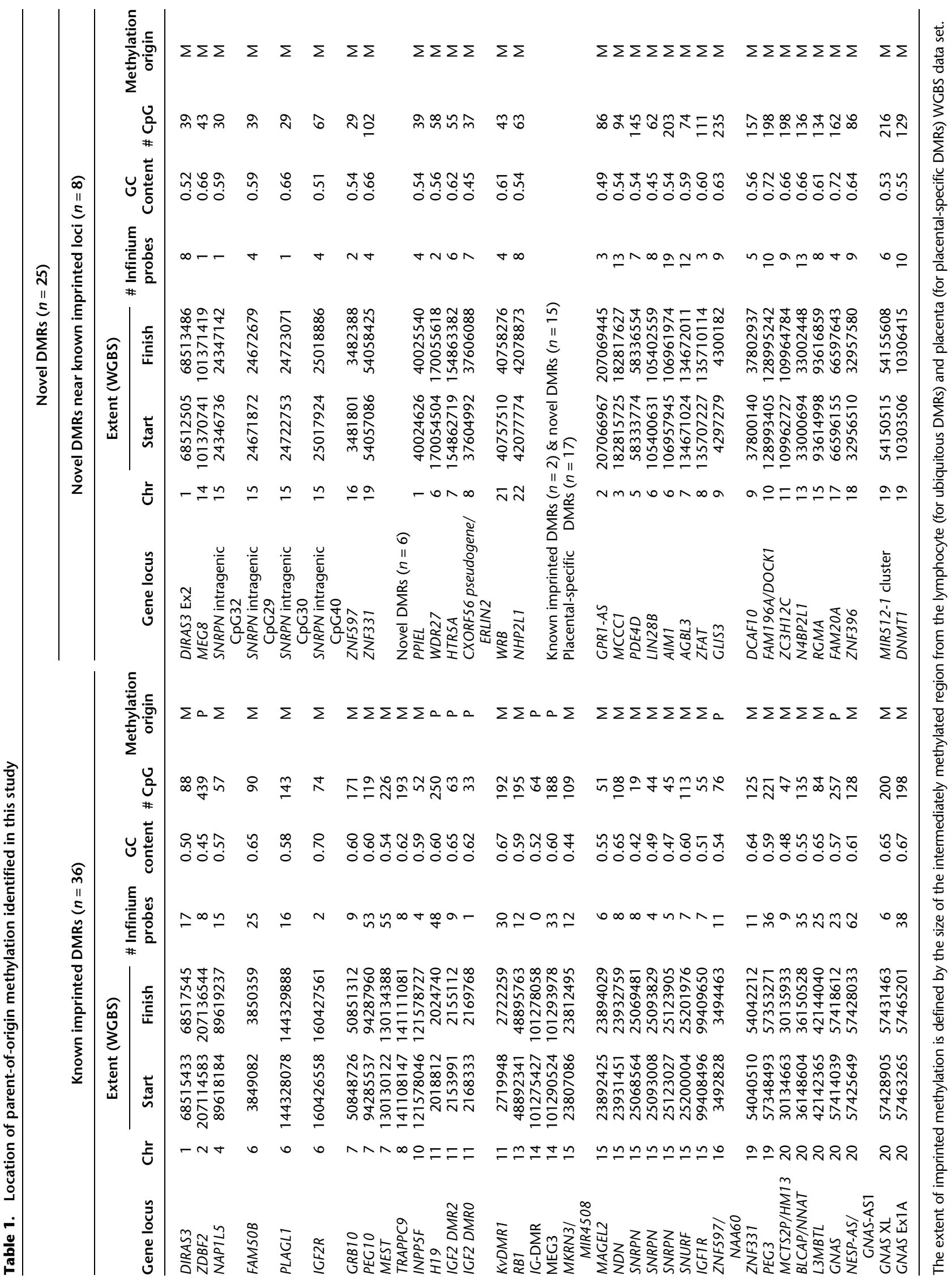


(A)

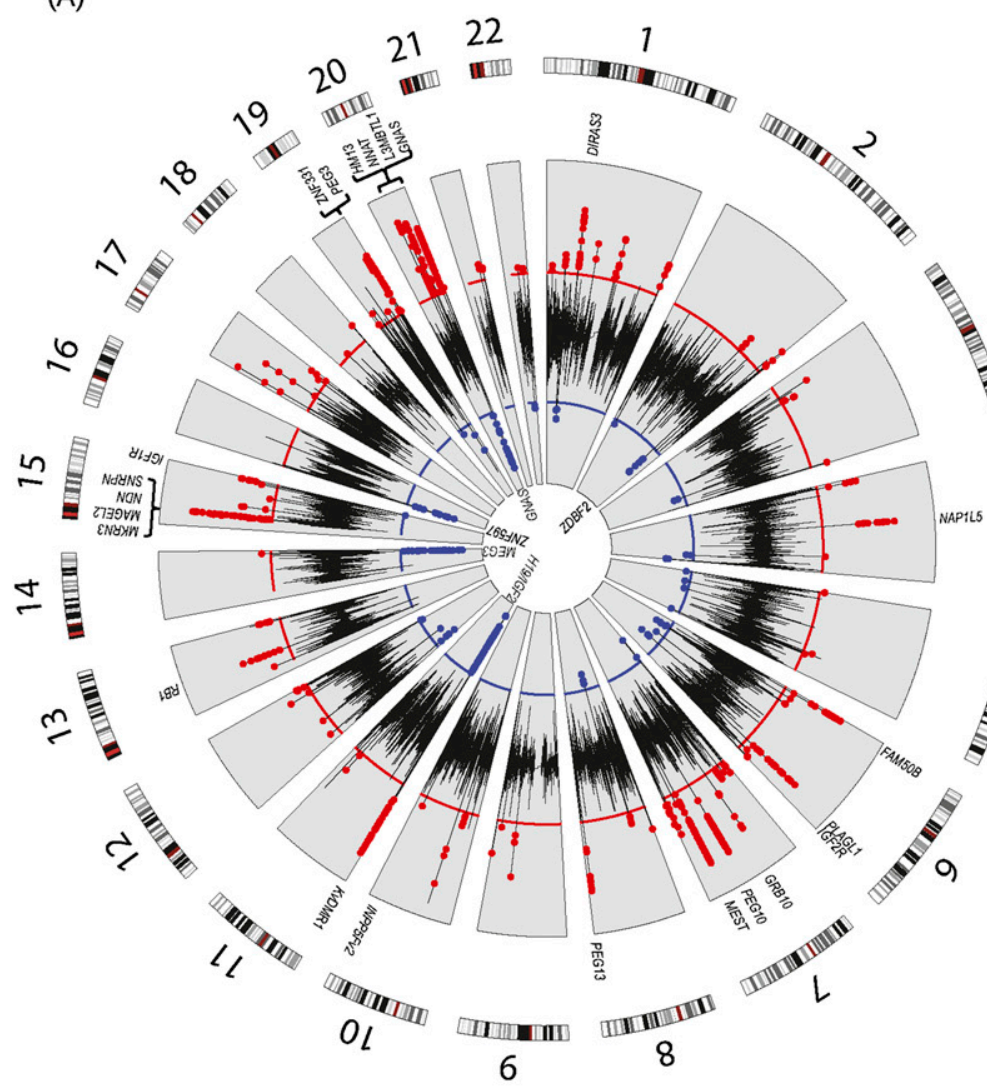

(B)

B)

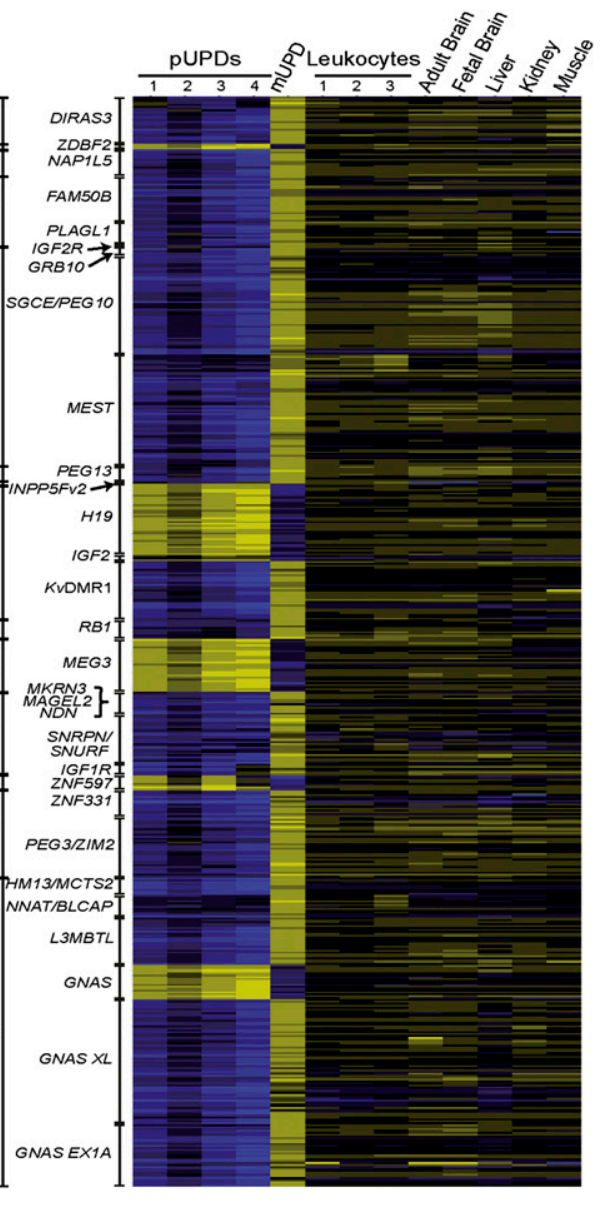

41

(C)
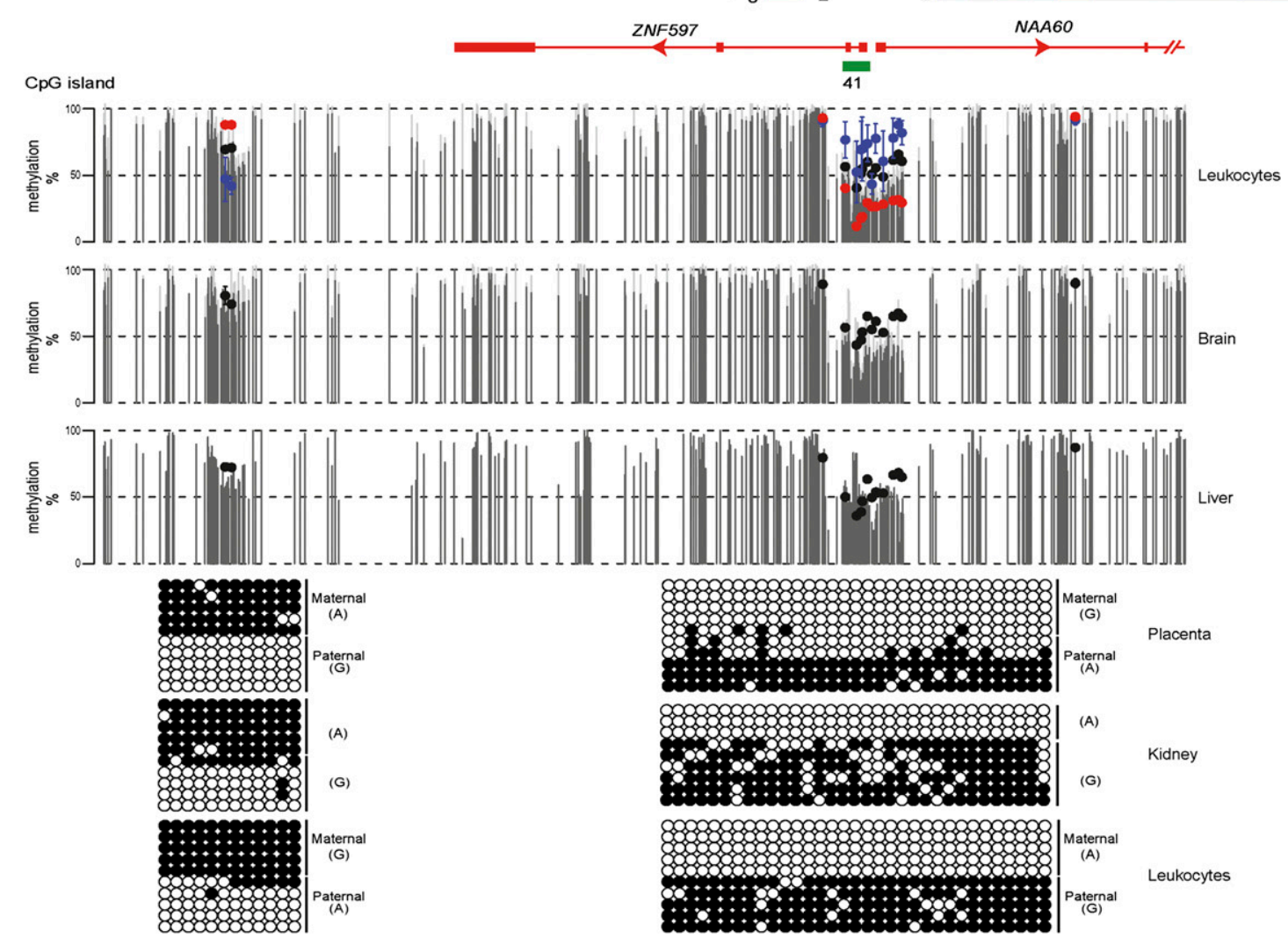

Figure 1. (Legend on next page) 
all regions and verified that six regions (PPIEL, WDR27, HTR5A, $W R B, N H P 2 L 1, E R L I N 2$ loci) are maternally methylated. The DMR we identify within intron 7 of ERLIN2 appears to be a retroinsertion of the CXorf56 pseudogene (also known as LOC728024) (Fig. 2B,C; Supplemental Fig. S3; Supplemental Table S1).

To confirm that parent-of-origin transcription occurs near these novel imprinted DMRs, we performed allelic RT-PCR in a panel of tissues with primers that discriminate major variant transcripts within each region. This revealed that the DMRs associated with WDR27, NHP2L1, and CXorf56 pseudogenes regulate allelic expression in an isoform-specific fashion (Fig. 2B,C; Supplemental Fig. S3; Supplemental Table S1). We detect monoallelic expression of a short alternatively polyadenylated ERLIN2 transcript which independently substantiates the observation that the generation of retrogenes, primarily from the X chromosome, is a common mechanism for generating imprinted loci (Wood et al. 2008; Kanber et al. 2009). Unfortunately, due to the lack of informative polymorphisms or expression in available heterozygous tissues, we could not perform allelic expression analysis for PPIEL, $H T R 5 A$, and $W R B$.

\section{Histone methylation of H3K4 and DNA methylation are enriched on opposing alleles at imprinted DMRs}

GC-rich sequences often coincide with enrichment of H3K4me3, which may act to protect them from de novo methylation (Thomson et al. 2010). The H3K4 demethylase KDM1B (previously known as AOF1) is required for appropriate establishment of maternal germline methylation for a subset of imprinted DMRs in mouse, suggesting that the presence of $\mathrm{H} 3 \mathrm{~K} 4$ methylation is refractory to DNA methylation deposition in the female germline (Ciccone et al. 2009). By comparing publicly available data sets for ChIP-seq for H3K4me3 and methylated DNA immunoprecipitation (meDIP-seq) from blood and brain, we observe co-enrichment of these opposing epigenetic marks at $89.5 \%$ of imprinted DMRs, consistent with differential active and repressive chromatin states on homologous chromosomes. For a limited number of informative regions, we were able to confirm $\mathrm{H} 3 \mathrm{~K} 4 \mathrm{me} 3$ precipitation on the unmethylated allele (Fig. 3C). In most cases, the methylation profile of maternally methylated DMRs is more closely related to the opposing $\mathrm{H} 3 \mathrm{~K} 4 \mathrm{me} 3$ profile rather than to the $\mathrm{CpG}$ density that classically defines CpG islands ( $>200 \mathrm{bp}$, GC content $>50 \%$, observed/expected ratio $>0.6$ ), with the exception of the GNAS-XL DMR. This maternally methylated region was thought to be a single regulatory unit; however, our WGBS and Infinium array data clearly show that it is two separate DMRs, partitioned by an $\sim 200$ bp interval of hypermethylation, with the centromeric GNAS-AS1 (previously known as NESP-AS) promoter showing coenrichment for H3K4me3 and DNA methylation, while the GNAS-XL side lacks this permissive histone modification (Fig. 3A).

Further interrogation of this data set identified two DMRs associated with multiple promoters with a gradient effect across the CpG-rich sequences. The GNAS/GNAS EX1A CpG island (CpG island 320 in Fig. 3A) is unmethylated on one side, coinciding with H3K4me3, whereas the other is differentially methylated with abundant H3K4me3 and meDIP reads. This pattern was also observed in the bidirectional HTR5A/HTR5A-AS1 promoter in brain (Fig. 3B), a tissue where these transcripts are most abundant.

\section{Tissue-specific dynamics of imprinted DMRs}

The WGBS analysis in leukocytes, brain, and liver confirmed that the extent of allelic methylation at the imprinted DMRs, as defined by the size of the intermediately methylated interval, is highly similar in these somatic tissues (Figs. 1, 4; Table 1). However, some regions were drastically different in the placenta.

By comparing the placental WGBS profile with Infinium $\beta$-values for placentae and hydatidiform moles, we observe that the DMRs associated with the maternally methylated PEG1O and the paternally methylated $H 19$ are significantly larger in placenta than in somatic tissues. Using standard bisulfite PCR and sequencing, we confirm that the somatically unmethylated SGCE promoter, immediately adjacent to the differentially methylated PEG10 promoter, is methylated on the maternal allele in placenta, while the maternal allele overlapping the $\mathrm{H} 19$ gene body is demethylated (Fig. 4B).

In addition to identifying extended DMRs in the placenta, we also observe complex tissue-specific methylation between somatic tissues and placenta. For example, the NNAT and GNAS-AS1 DMRs, which are maternally methylated in somatic tissue, exhibit hypermethylation in both placenta and hydatidiform mole. Subsequent bisulfite PCR confirmed that these regions are fully methylated in the placenta (Supplemental Fig. S4). Methylation profiling at the MIR512-1 cluster (also known as C19MC)-ZNF331 locus on chromosome 19 has previously disclosed that the promoter of the pri-miRNA for this miRNA cluster is maternally methylated in placenta, but fully methylated in somatic tissues (Noguer-Dance et al. 2010). We confirm that the MIR512-1 DMR is unmethylated in hydatidiform moles compared to the partially methylated profile in placenta, with placental WGBS revealing that the DMR is $\sim 5 \mathrm{~kb}$ in size, incorporating the promoter $\mathrm{CpG}$ island (CpG island 86 in Fig. 4C). However, we notice that the $\mathrm{CpG}$ island (CpG island 83 in Fig. 3C) associated with ZNF331 isoform-3 is hypermethylated on both parental alleles in placenta but is a maternally methylated DMR in somatic tissues. These methylation states dictate complex allelic expression at this locus, with restricted placental-specific paternal expression of the MIR512-1 pri-miRNA, which does not extend to the MIR371/2 cluster, and reciprocal imprinting of ZNF331 (Fig. 4C; Supplemental Table S2).

\section{Novel placental-specific DMRs associated with paternally expressed transcripts}

Based on the complex methylation profiles described above, we next investigated if more unknown imprinted DMRs exist solely in

Figure 1. Identification of known imprinted DMRs on the Infinium array platform. (A) Circular karyotype showing the difference of methylation for three consecutive probes for reciprocal UPD leukocyte samples. Red dots indicate a minimal difference of 0.3 in Infinium probe $\beta$-values $(>30 \%$ absolute methylation value) for regions with maternal methylation, and blue dots indicate the same for paternal methylation. Known DMRs are indicated. (B) Heat map of the Infinium probes located within known imprinted DMRs in reciprocal genome-wide UPD samples and various somatic tissues. (C) WGBS and Infinium array methylation profiles of the ZNF597 locus with bisulfite PCR confirmation of the novel maternally methylated DMR and its position in relation to the somatic paternally methylated promoter region. Vertical gray lines in the WGBS tracks represent the mean methylation value for individual CpG dinucleotides calculated from multiple data sets, with the light gray lines representing the mean + standard deviation. Infinium methylation values for normal tissues are represented by black dots, with values for the genome-wide UPDs (average pUPD in blue and mUPD in red) superimposed on the leukocyte methylation track. The error bars associated with the Infinium array probes represent the standard deviation of multiple biological samples. The PCR confirmation in placenta, kidney, and leukocyte-derived DNA was performed on heterozygous samples. Each circle represents a single CpG dinucleotide on a DNA strand. $(\bullet)$ Methylated cytosine, $(\bigcirc)$ unmethylated cytosine. Each row corresponds to an individual cloned sequence.

\section{Genome Research}


(A)

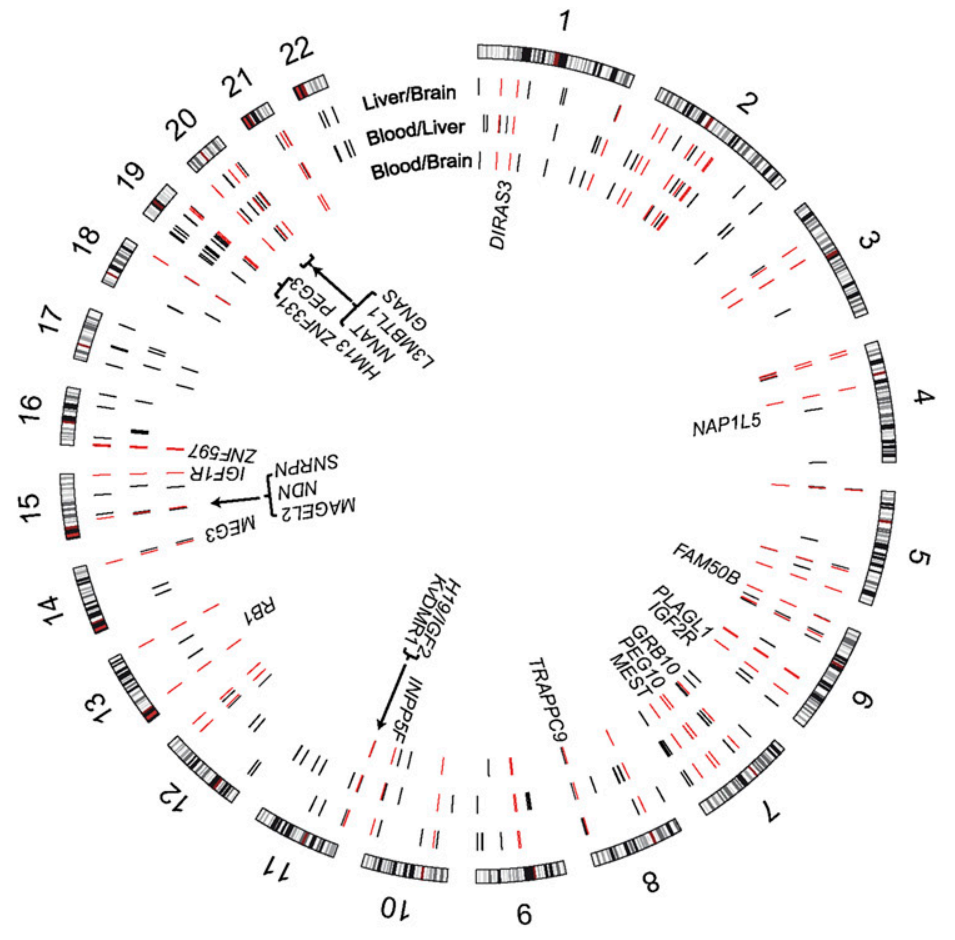

(B)
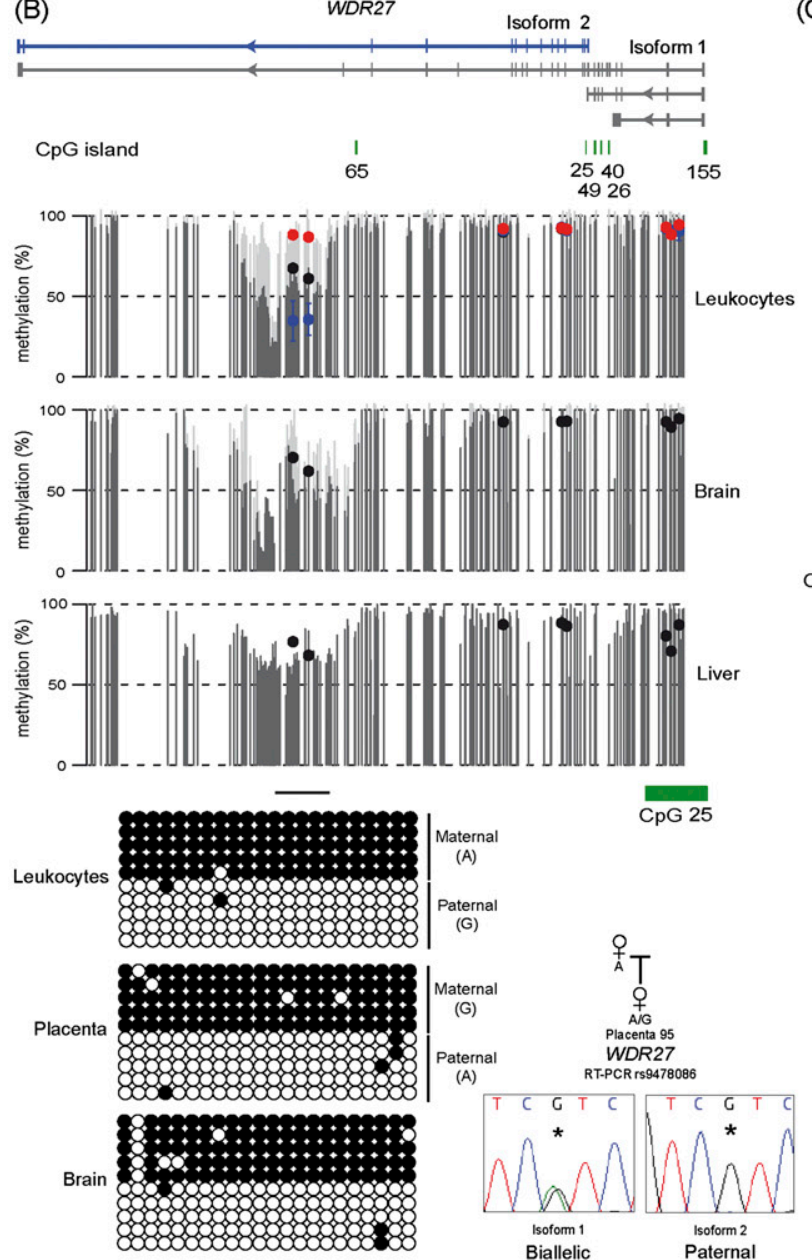

Figure 2. (Legend on next page)

(C)

Brain
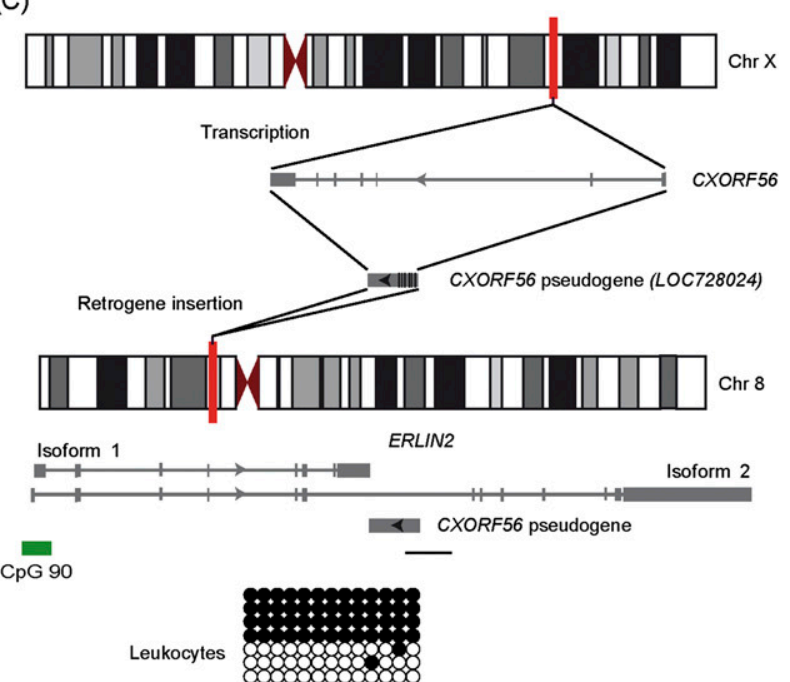

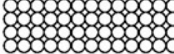
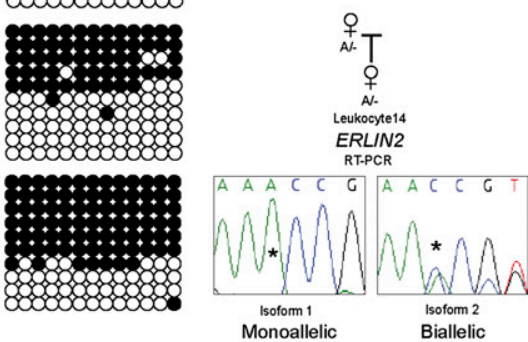

Genome Research 559 www.genome.org 
placental tissues, as highlighted by the MIR512-1 and GPR1-AS DMRs (Noguer-Dance et al. 2010; Kobayashi et al. 2013).

We performed a screen for partially methylated regions present solely in our placenta WGBS data set using our sliding window approach $(0.25<$ mean of $25 \mathrm{CpG} \pm 2 \mathrm{SD}<0.75)$. This identified 722 windows, of which 520 mapped to CpG islands. These results confirm that placental-derived DNA is significantly less methylated when compared to other tissues (Schroeder et al. 2013) and that this genome-wide lower methylation is not restricted to repetitive elements as previously described (Ehrlich et al. 1982; Fuke et al. 2004), but occurs across a large portion of the genome.

Of these partially methylated placenta domains identified by WGBS, 44 regions were $\sim 50 \%$ methylated in placenta, with extreme methylation in hydatidiform moles using the Infinium array (average $\beta$-value for three consecutive probes $>0.8$ indicative of paternal methylation or $<0.2$ indicative of maternal methylation), and showed no evidence of allelic methylation in somatic tissues. Using standard bisulfite PCR, we assessed the allelic methylation profile of all regions in placental DNA samples. This revealed that the promoters of N4BP2L1, DCAF10, PDE4D, FAM196A, RGMA, AGBL3, MCCC1, ZC3H12C, DNMT1, AIM1, ZNF396, FAM20A, GLIS3, and LIN28B are methylated on the maternal allele (Fig. 4D; Supplemental Fig. S5; Supplemental Table S2). In addition, we identified a $2.8-\mathrm{kb}$ region of intermediate methylation overlapping an alternative promoter of the paternally expressed ZFAT gene in the placental WGBS data set (Supplemental Fig. S5). Using allelicspecific bisulfite PCR, we confirm that the methylation is confined to the maternal chromosome at this locus. To determine if these regions of maternal methylation influence transcription, allelic RT-PCR experiments were carried out. Paternal expression of eight of these genes was verified, with biallelic expression in somatic tissues (Fig. 4D; Supplemental Fig. S5; Supplemental Table S2) consistent with recent allelic expression screens in term placenta (Yuen et al. 2011; Barbaux et al. 2012).

\section{Mammalian conservation of novel imprinted domains}

To determine if the previously unrecognized imprinted domains are conserved throughout evolution, we assessed their allelic methylation and expression in mice, using a reciprocal cross between mouse strains. Bisulfite PCR targeting of orthologous regions failed to identify evidence of differential methylation in embryonic day E9.5-14.5 embryos or extra-embryonic tissues. Subsequent allelic RT-PCRs revealed that all murine transcripts orthologous to the novel ubiquitous and placental-specific imprinted transcripts are equally expressed from both parental alleles when detected (Supplemental Figs. S6, S7). This suggests that these new imprinted domains arose less than $\sim 80$ million years ago after the divergence of mice and humans or that selection pressures over this period have resulted in a loss of imprinted regulation of these genes in mice. It has been previously reported that imprinting in the placenta dif- fers between human and mouse, mainly due to the lack of imprinting of genes which require repressive histone modifications for allelic silencing in humans (Monk et al. 2006). Contrary to previous reports, our results show that humans have evolved more loci subject to this form of transcriptional regulation in placenta, due to the evolutionary acquisition of loci with parent-of-origin methylation. This is endorsed by the low discovery rate of novel imprinted transcripts in RNA-seq screens of mouse placenta (Okae et al. 2011).

\section{Differential methylation at ubiquitously imprinted loci and placental-specific domains may differ in their gametic origin}

An essential step toward understanding the establishment of the germline imprint signal is to determine if the parent-of-origin methylation observed in somatic tissues is derived from the germline. Determining the methylation profiles in human gametes and during the early preimplantation stages of embryonic development is technically and ethically challenging. To circumvent these difficulties, we have used a combination of mature gametes and in vitro models to represent human gametes of both sexes and preimplantation embryos. For analysis during gametogenesis in males, we used mature sperm. We compared publicly available WGBS data sets from sperm and human embryonic stem (hES) cells that represent the inner cell mass of the blastocysts (Lister et al. 2009; Molaro et al. 2011) with our own Infinium array profiles for sperm, parthenote-derived hES cell lines (phES), and hES cell lines generated from both six-cell blastomeres (Val10B) and the inner cell mass of blastocysts (SHEF cell lines). Despite the phES cell lines having undergone reprogramming during blastocyst development, they have previously been shown to retain maternal hypermethylation at the limited imprinted loci assessed, suggesting that they are ideal surrogates for assessing the methylation profiles of imprinted DMRs in mature oocytes (Mai et al. 2007; Harness et al. 2011).

A comparison of Infinium $\beta$-values between sperm and phES cells for the human sequences orthologous to the mouse germline DMRs (Kobayashi et al. 2012) revealed that 19/22 are conserved. The novel ubiquitous DMRs we identify are also hypermethylated in phES cells and unmethylated in sperm, suggesting that the majority of imprinted DMRs, with the exception of $I G F 1 R$, are primarily marked in the gametes (Fig. 5A; Supplemental Fig. S8). In addition, we confirm that the IG-DMR within the chromosome 14 domain is $>80 \%-90 \%$ methylated in the sperm WGBS data set, in line with previous reports (Geuns et al. 2007). We were particularly intrigued to observe that all placental-specific DMRs, with the exception of ZFAT, GPR1-AS, and MIR512-1, do not inherit methylation from the gametes and are devoid of methylation in hES cells (Fig. 5A). These data provide preliminary evidence to suggest that, following gametogenesis, parental alleles at some loci retain a nonequivalency that is not associated with DNA methylation.

Figure 2. Identification and characterization of allelic methylation and expression of novel imprinted loci. Circular karyotype showing the position of common regions of intermediate methylation in the leukocyte, brain, and liver WGBS data sets, as identified using a 25 CpC sliding window approach $(0.25<$ mean $\pm 1.5 \mathrm{SD}<0.75)$. Red ticks represent sites of intermediate methylation common to all tissues, whereas black ticks identify those present in only one or two pairwise comparisons. The position of known imprinted DMRs are shown. (B) Identification of a novel maternally methylated DMR within the WDR27 locus by WGBS and Infinium array analysis. Vertical gray lines in the WGBS tracks represent the mean methylation value for individual CpG dinucleotides calculated from multiple data sets, with the light gray lines representing the mean + standard deviation. Infinium methylation values for normal tissues are represented by black dots, with values for the genome-wide UPDs (average pUPD in blue and mUPD in red) superimposed on the leukocyte methylation track. The DMR was confirmed using standard bisulfite PCR on heterozygous DNA samples and orchestrates paternal expression of WDR27 isoform 2. The asterisk $\left(^{*}\right)$ in the sequence traces shows the position of the polymorphic base. (C) Imprinting of ERLIN2 isoform 1 in leukocytes as a consequence of the retrotransposition of the $\mathrm{X}$ chromosome-derived CXorf56 pseudogene into the locus.

\section{Genome Research}

www.genome.org 
(A)

MIR296/MIR298 GNAS-AS1
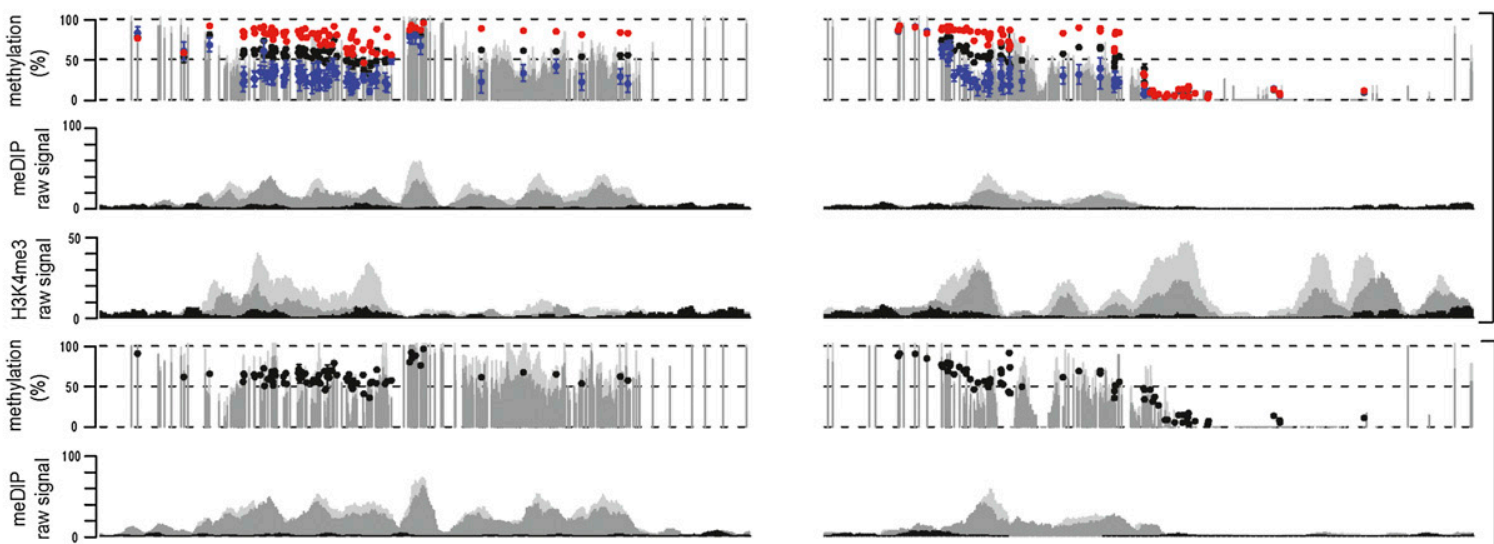

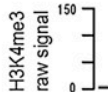

CpG island
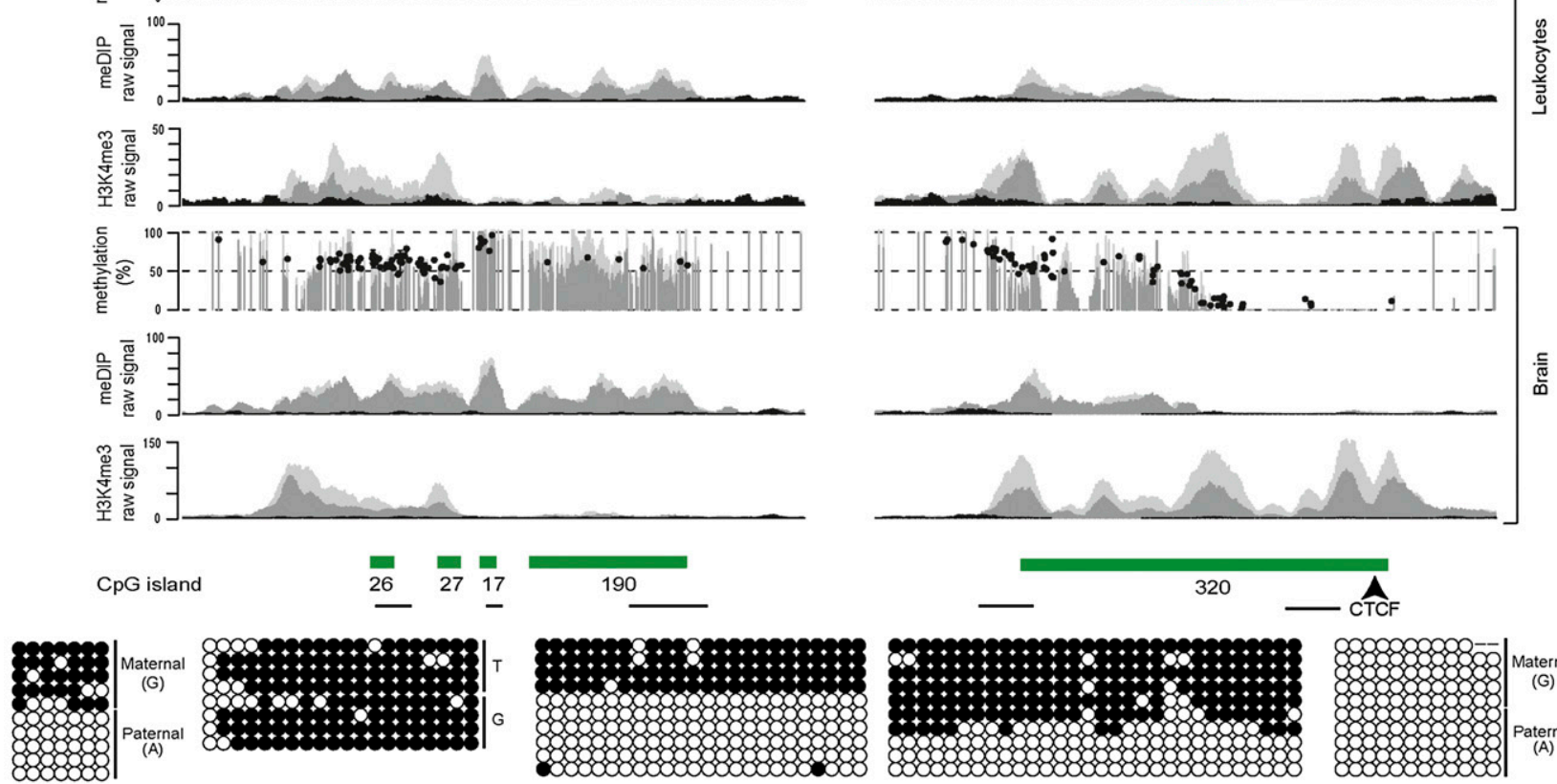

(B)
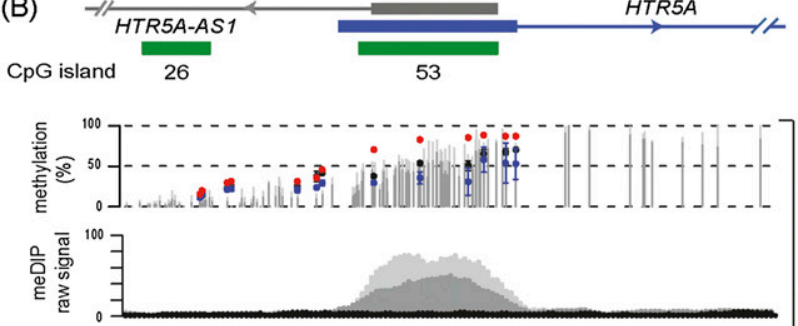

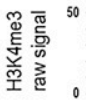



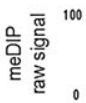

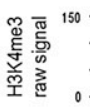

CpG island

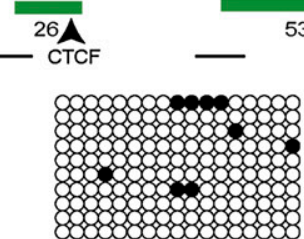

53

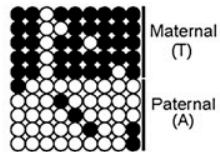

(C)

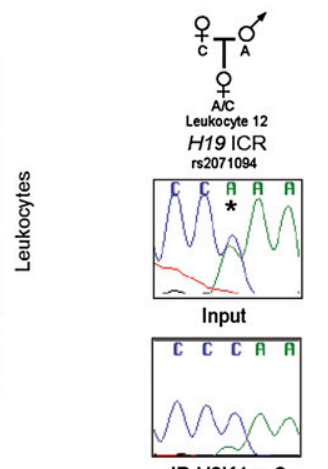

IP-H3K4me3

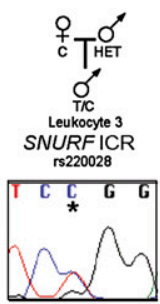

Input

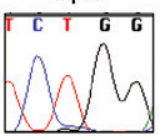

IP-H3K4me3

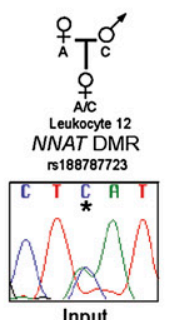

Input

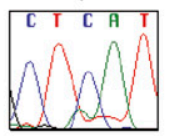

IP-H3K4me3
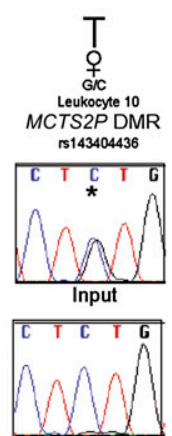

IP-H3K4me3

Figure 3. H3K4me3 chromatin profile and DNA methylation at imprinted loci. (A) Map of the human GNAS locus on chromosome 20 with the H3K4me3 and meDIP signatures in brain and leukocytes at the DMRs identified in the WGBS and Infinium array analysis. Infinium methylation values for normal leukocytes (black dots), with values for the genome-wide PUPD (blue) and mUPD (red) superimposed on the leukocyte WGBS track. Similarly, Infinium methylation values for two normal brain samples are shown as black and gray dots. The light and dark gray peaks in the meDIP and ChIP-seg panels represent two independent biological replicates compared to input (black peaks). The bars under the CpG islands, as identified in the UCSC Genome Browser, show the location of the bisulfite PCR amplicons. (B) The gradient DMR identified at the HTR5A promoter. The samples used for the WGBS, Infinium array, and ChIP are the same as in $A$. The independent methylation pattern on either side of the bidirectional promoter interval was confirmed using standard bisulfite PCR and sequencing. (C) Allelic ChIP for H3K4me3 reveals predominant enrichment of this histone modification on the unmethylated allele of the H19 ICR, SNURF ICR, NNAT, and MCTS2P DMRs. The asterisk $\left(^{*}\right)$ in the sequence traces shows the position of the polymorphic base. 
(A)

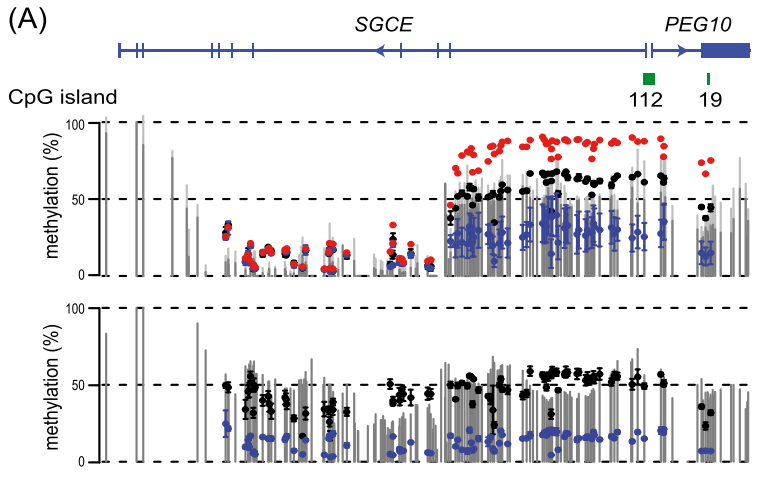

CpG island

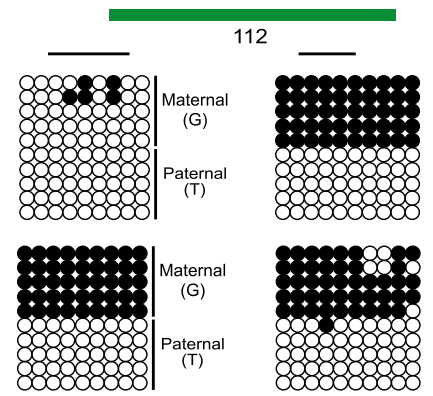

(C)

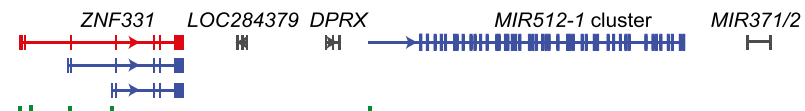

$\begin{array}{lccc}\text { CpG island } & \text { I' } & \text { ' } & \text { I } \\ & 45 & 100 & 83\end{array}$
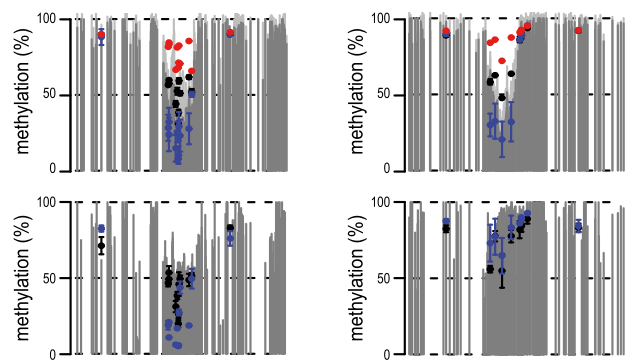

CpG island 100

83

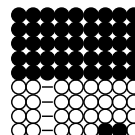

(C)

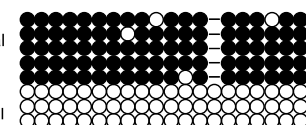

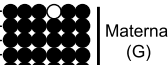

Maternal
$(G)$
Paternal

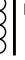

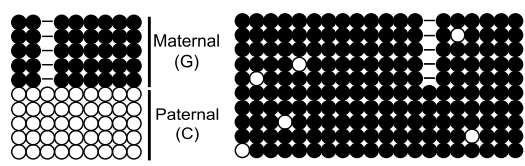

${ }_{G}^{\circ} T_{A G}^{\sigma}$

$\underset{\substack{+ \\ \text { A/G }}}{+}$

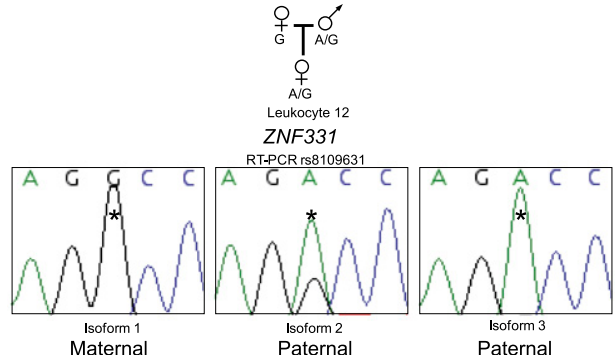

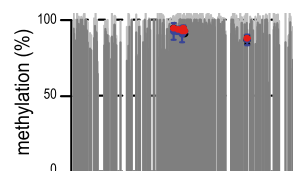

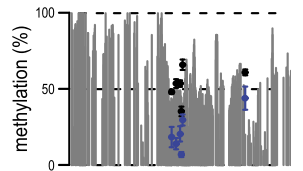

86
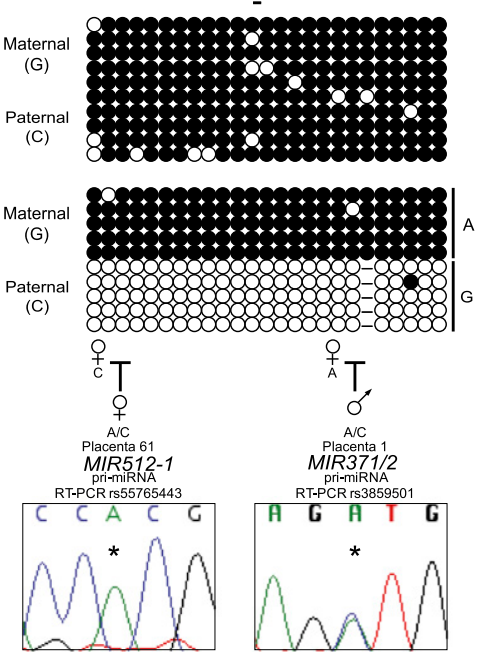

Paternal

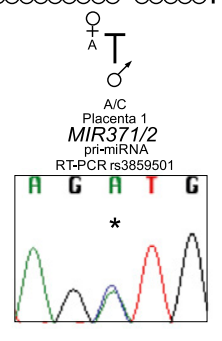

Biallelic

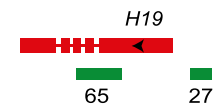

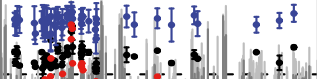

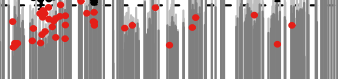

Leukocytes
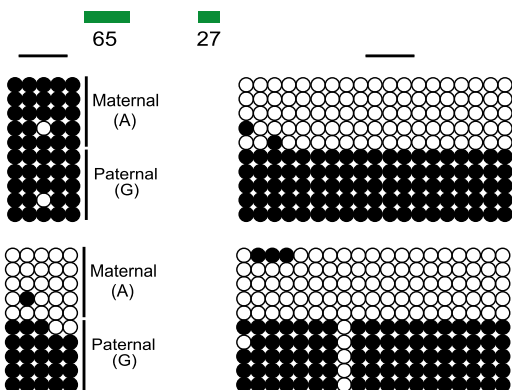

(D)

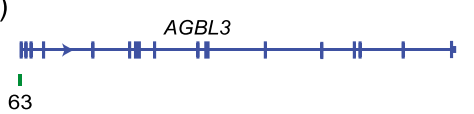

Figure 4. (Legend on next page)
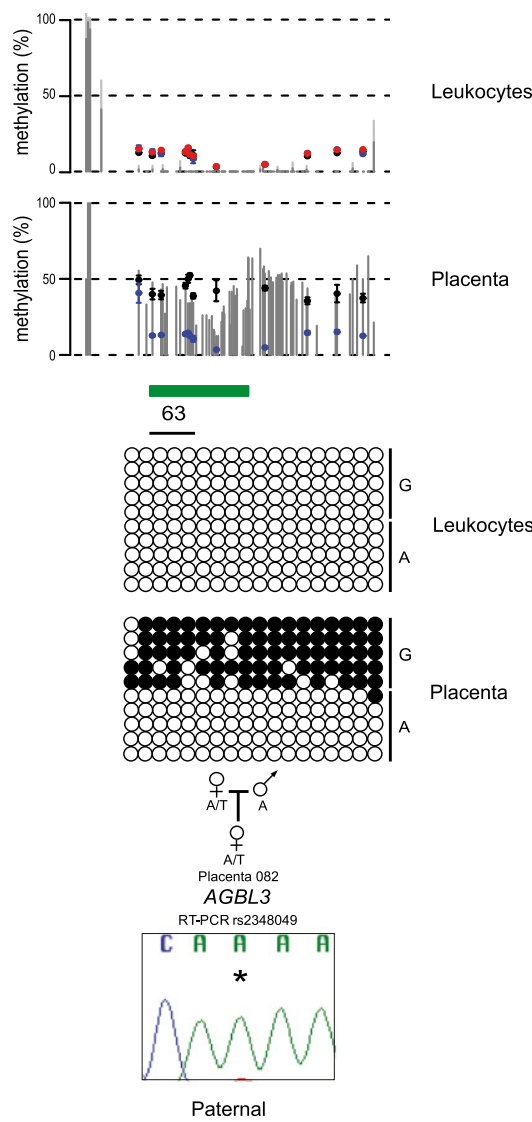
Therefore, in the female germline, as represented by the phES cells, a subset of imprinted loci retain their identity in the absence of methylation, suggesting that additional epigenetic mechanisms mark these regions for maternal methylation during trophoblast differentiation (Fig. 5A).

For the majority of DMRs for which allelic methylation was observed in the somatic tissues $(80 \%)$, the genomic interval showing methylation differences between sperm and phES cells is larger than the allelic DMRs in hES cells and somatic tissues (Fig. $5 \mathrm{~B}, \mathrm{C})$. In the case of maternally methylated DMRs, we observe that these regions are flanked by fully methylated intervals in both gametes, and that these DMRs are observed as regions devoid of methylation in the sperm genome. Interrogation of ChIP-seq data sets for nucleosomes containing the histone modifications revealed that the majority of unmethylated DMR regions in sperm are enriched for H3K4me3 containing nucleosome fractions. Our analysis indicates that the size of the unmethylated region in sperm is therefore associated with nucleosome occupancy, rather than protamines. Notably, the maternally methylated germline DMR overlapping the NNAT promoter is $\sim 4 \mathrm{~kb}$, as defined by full methylation in phES cell and the H3K4me3 enriched DNA unmethylated region in sperm. This region contracts to an $\sim 1.5-\mathrm{kb}$ region of maternal methylation after preimplantation reprogramming as represented by blastocyst-derived hES cells and somatic tissue profiles (Fig. 5C; Supplemental Fig. S9A showing the contraction at the NAP1L5 locus). Such resizings are also observed in mouse (Tomizawa et al. 2011), suggesting that imprinted DMRs are not totally protected from genome-wide demethylation during the oocyte to embryo transition. We speculate that the larger regions of differential methylation dictated by the gametes, in combination with protective factors, ensure that they survive reprogramming.

In addition, we also observe other subtle differences in germline-derived methylation profiles. For example, the two sides of the GNAS-XL DMR that we show to have independent H3K4 methylation profiles from each other behave differently in the germline, with the GNAS-AS1 side being a somatic DMR only, but the GNAS-XL side being methylated in phES cells and hypomethylated in sperm (Supplemental Fig. S4). Lastly, we identified a dynamic relocalization of methylation at the FAM5OB DMR during preimplantation development. The $1.2-\mathrm{kb}$ promoter of this imprinted retrogene is methylated on the maternal allele in somatic tissues but is completely unmethylated in phES cells and hES cells derived from six-cell embryos, and has been shown to be unmethylated in sperm (Nakabayashi et al. 2011). However, we do find that allelic methylation is conferred during preimplantation development, at a point between the six-cell stage and blastocyst development. In fact, the $\sim 1$-kb regions flanking the promoter (labeled 1 and 3 in Supplemental Fig. S9B) show strongly opposing methylation profiles, with the sperm being unmethylated and phES cells methylated, which then become fully methylated on both alleles immediately after fertilization, leaving allelic methylation over the promoter itself.

\section{Discussion}

Differentially methylated regions between the parental alleles are essential for genomic imprinting and development. In this study, we have performed a comprehensive survey of methylation in various human tissues, uncovering all known imprinted DMRs as well as 21 novel loci, which we demonstrate wherever possible regulate imprinted transcription. Our present work demonstrates that the human genome contains a significantly larger number of regions of parent-of-origin methylation than previously thought. The identification of imprinted domains has traditionally been performed in mouse by utilizing gynogenetic and androgenetic embryos, mice harboring regions of uniparental disomies, or highly polymorphic inbred strains (Cooper and Constância 2010). These embryos have been subjected to expression-based screens, including RNA-seq (Gregg et al. 2010; Okae et al. 2011), and genomewide methylation techniques (Hayashizaki et al. 1994; Kelsey et al. 1999; Hiura et al. 2010). By relying on the confirmation of the evolutionarily conserved expression of the human orthologs, imprinted genes specific to higher primates and humans would have been missed. We have utilized high-throughput bisulfite analysis from in vitro models of gametes and early embryos, and somatic and placental DNA, to characterize the developmental dynamics of imprinted methylation coupled with allelic expression analysis of nearby transcripts. This analysis reveals that 30 regions of parentally inherited differential methylation are observed in humans but not mice. Conversely, we also show that the DMRs associated with Cdkn1c, Rasgrf1, the Igf2r promoter, Impact, Slc38a4, and Zrsr1 (previously known as U2af1-rs1) imprinted transcripts in mouse do not exhibit allelic methylation in humans (Xie et al. 2012).

Recently, a novel mechanism has been described in which differences in germline methylation can give rise to tissue-specific DMRs in mouse (Proudhon et al. 2012). The Cdh15 DMR inherits methylation from the oocyte and maintains this parental allelic methylation during in utero development and in adults, with the exception that the paternal allele gains methylation in various brain regions. Therefore, the intragenic Cdh15 DMR is conserved during adulthood, but in a tissue-specific manner. In humans, the CDH15 locus does not exhibit allelic DNA methylation in any tissue (data not shown), suggesting that this tissue-specific methylation profile might be limited to mice. We cannot rule out the existence of temporally regulated tissue-specific imprinted DMR in humans, since our samples were derived from adults, and therefore any imprinted DMRs specific to the fetal period would be missed.

Our study reveals the power of combining WGBS and Infinium HumanMethylation450 BeadChip arrays to identify novel imprinted DMRs. We have previously combined reciprocal genomewide UPD samples and the Infinium HumanMethylation27

Figure 4. The methylation profiles of imprinted loci in placenta compared to somatic tissues. The placenta- and leukocyte-derived WGBS and Infinium array profiles at the $(A)$ PEG 10 and $(B) H 19$ loci. Infinium methylation values for normal leukocytes (black dots), with values for the genome-wide pUPD (blue) and mUPD (red) superimposed on the leukocyte WGBS track. Similarly, Infinium methylation values for normal placenta (black dots) and hydatidiform mole (blue dots) are overlaid on the placental WGBS track. The error bars associated with the Infinium array probes represent the standard deviation of multiple biological samples. Bisulfite PCR analysis was used to confirm the tissue-specific methylation profiles. (C) Complex tissue-specific allelic methylation and expression patterns at the ZNF331-MIR512 cluster locus on chromosome 19. The ZNF331 sequence traces represent the RT-PCR products from leukocytes, whereas both the MIR512-1 cluster and MIR371/2 are from placenta. (D) A placental-specific imprinted DMR identified using the placentaderived WGBS and Infinium array data sets. The methylation profiles were confirmed using standard bisulfite PCR on heterozygous DNA samples with allelic RT-PCR performed on placental biopsies. The results confirm that the region of maternal methylation overlapping the $A G B L 3$ promoter dictates paternal expression of this gene in placenta. 


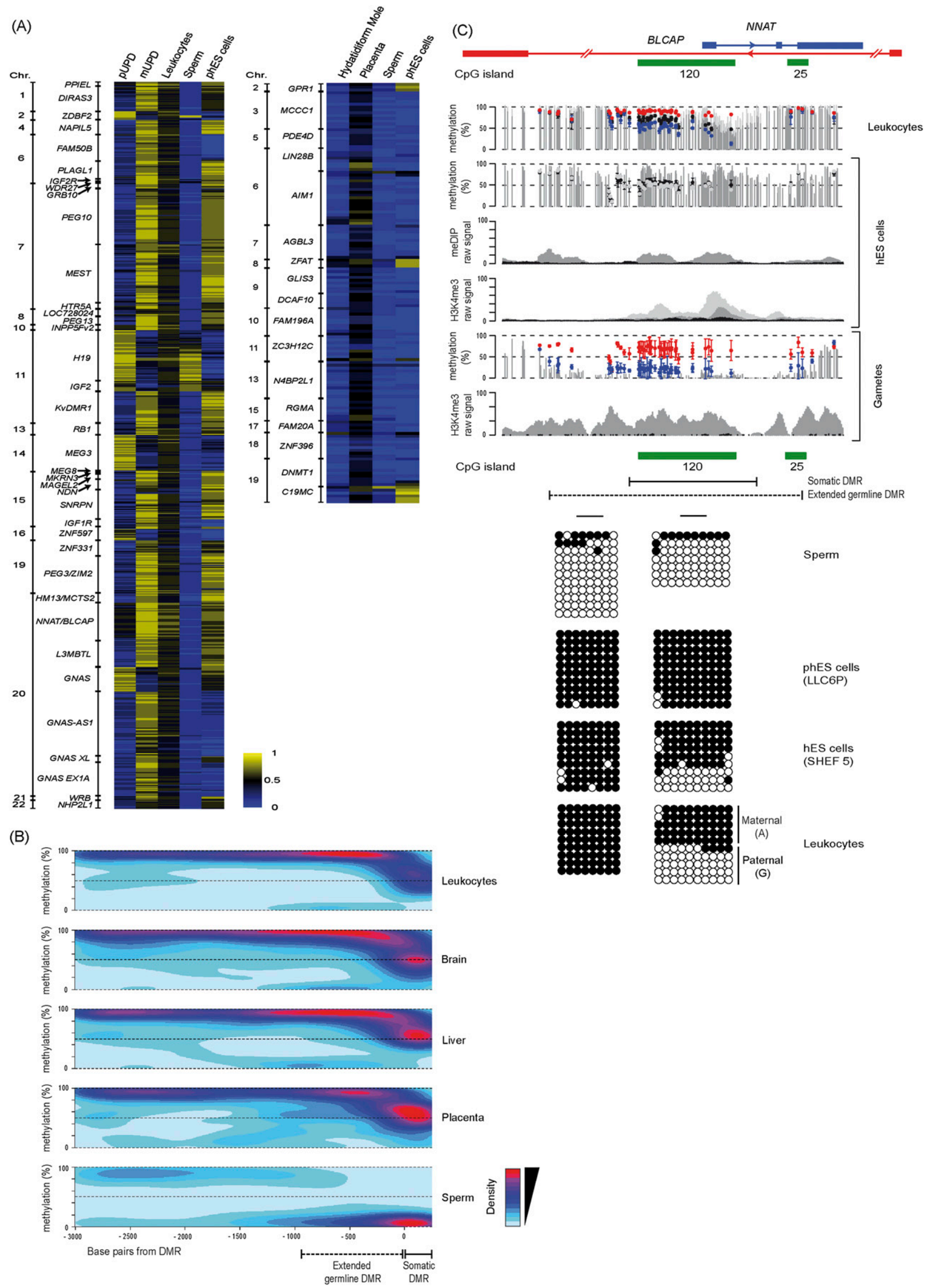

Figure 5. (Legend on next page) 
BeadChip arrays to identify imprinted loci (Nakabayashi et al. 2011). All new regions of ubiquitous imprinted methylation identified in the current screen are associated predominantly with type II Infinium probes and were not present on previous array platforms. Of the placental-specific DMRs, only those associated with DNMT1, AIM1, and MCCC1 have been previously described (Yuen et al. 2011; Das et al. 2013). Intriguingly, the somatic promoter of Dnmt1 is differentially methylated between sperm and oocytes but is lost during preimplantation development (Smallwood et al. 2011; Kobayashi et al. 2012). Two of these placental-specific DMRs are associated with type I Infinium probes and were previously discovered using the Infinium HumanMethylation27 BeadChip arrays with DNA derived from diandric and digynic triploid placental samples (Yuen et al. 2011).

Our data provide the first direct evidence in humans that the differential methylation associated with imprinted genes is dynamically regulated upon fusion of the gametes at fertilization. Most maternally methylated DMRs are surrounded by regions of complete methylation in both gametes, and as in mice, the DMRs are clearly observed as unmethylated islands in the sperm genome. These unmethylated intervals are often more extensive in sperm compared to somatic tissues, suggesting that resizing occurs during embryonic transition. It was recently reported that nucleosomes are retained at specific functional regions in sperm chromatin and are refractory to protamine exchange (Hammoud et al. 2009). These sperm-derived histones are enriched for H3K4me3, a permissive modification that is mutually exclusive with DNA methylation, implicating these $\mathrm{H} 3 \mathrm{~K} 4 \mathrm{me} 3$ regions in the maintenance of the unmethylated state in the male germline.

Imprints are distinguishable from other forms of gametic methylation as they survive the reprogramming that initiates immediately upon fertilization (Smallwood et al. 2011; Kobayashi et al. 2013; Proudhon et al. 2012). By comparing the profiles of sperm, phES, and conventional hES cells along with somatic tissues, we present evidence that most maternally methylated DMRs are not completely refractory to reprogramming, as highlighted by the substantial resizing of the paternally derived unmethylated alleles. These data are consistent with the notion that the cores of imprinted DMRs are protected from Tet-associated demethylation by recruiting heterochromatic factors such as ZFP57 and DPPA3 (also known as STELLA or PGC7) (Nakamura et al. 2007; Li et al. 2008). Similar mechanisms could also act to protect the core of the unmethylated paternal alleles from methylation.

A search for the mouse ZFP57 recognition sequence (TGCC ${ }^{\text {met }}$ GC) identified numerous binding sites within the ubiquitous imprinted DMRs that may be involved in protecting methylation during preimplantation reprogramming (Quenneville et al. 2011). It is currently unknown if this hexonucleotide motif is bound by ZFP57 in human cells, but patients with mutated ZFP57 lack DNA binding capacity in in vitro EMSA studies (Baglivo et al. 2013).
There are significantly fewer ZFP57 sequence motifs in the placental-specific DMRs compared to the ubiquitous DMRs that inherit methylation from the germline $(P<0.05$, Student's $t$-test), with 14/17 placental-specific DMRs being unmethylated and not associated with H3K9me3 in hES cells (Supplemental Fig. S10). These data further support our hypothesis that a novel imprinting mechanism occurs in the placenta, which is one of the first examples of methylation-independent epigenetic inheritance in mammals. In support of our observations, Park and colleagues (Park et al. 2004) generated a H19 ICR knock-in at the Afp locus which was de novo methylated around gastrulation, implying that H19 ICR is differentially marked in the gametes by a mechanism other than methylation. However, it is unknown if this mechanism also occurs at the endogenous $H 19$ locus. In our examples of placental-specific DMRs, the epigenetic mark inherited from the oocyte is currently unknown, but must be recognized by the de novo methylation machinery during early trophoblast differentiation, since we observe maternal methylation in term placenta. Certain histone methylation states are reported to recruit DNMTs (Dhayalan et al. 2010; Zhang et al. 2010). Since various posttranslational modifications of histone tails have been shown to be present at imprinted loci, specifically in the placenta independent of DNA methylation (Umlauf et al. 2004; Monk et al. 2006), we are led to suggest one inviting hypothesis: A histone modification confers the "imprint" at these novel placental-specific imprinted loci. Alternatively, the DNMTs may be recruited to these loci by a specific, yet to be identified, transcription factor expressed during early trophoblast differentiation.

In line with other well-characterized imprinted genes in the placenta, the placental-specific imprinted transcripts may also exert supply-and-demand forces between the developing fetus and mother, ultimately influencing fetal adaptation in utero, which if disrupted may have long-term consequences on health many decades after delivery (Constância et al. 2004). Our observation of imprinting of the somatic promoter of DNMT1 in placenta may therefore assist in this process. In addition, numerous studies have also suggested that children born as a result of assisted reproductive technologies (ART), including ovarian stimulation, in vitro fertilization, and intra-cytoplasmic sperm injections, have a higher risk of diseases with epigenetic etiologies, including imprinting disorders (Amor and Halliday 2008). In a clinical context, the placenta-specific imprinted loci may be prone to epigenetic instability during ART, as the first differentiation step that results in the trophectoderm occurs when the developing blastocysts are in culture.

By utilizing genome-wide methylation profiling at base-pair resolution, we have catalogued regions of parentally inherited methylation associated with imprinted regions and highlighted all differences between somatic and placental tissues. Further studies of these loci will provide insight into the causes of epigenetic ab-

Figure 5. Methylation in gametes, hES cells, and somatic tissues. ( $A$ ) Heat maps for Infinium probes mapping within all ubiquitous (left) and placentalspecific (right) imprinted DMRs in sperm and phES cells reveal the germline acquisition of methylation. (B) Methylation contour plots from WGBS data sets for all maternally methylated DMRs reveal that the extent of the intermediately methylated regions associated with imprinted DMRs are extremely consistent between somatic tissues and significantly larger in sperm. (C) Methylation profiles at the NNAT DMR determined by WGBS, Infinium array, and meDIP-seq data sets in leukocytes, sperm, phES cells, and hES cells, along with the H3K4me 3 ChIP-seq reads for hES cells and sperm. The gray and black dots in the second panel represent Infinium probe methylation in hES cell lines derived from six-cell blastomeres (Val10B) and blastocytes (SHEF5), respectively. The gametic WGBS methylation profile is derived from sperm, with Infinium probe methylation values for sperm and phES cells represented by blue and red dots. The graphic shows the extent of the differentially methylated regions in somatic tissues and between sperm and phES cells. The error bars associated with the Infinium array probes represent the standard deviation of the two sperm samples and four independent phES cell lines. The H3K4me3 ChIP-seq data is from sperm. The methylation profiles were confirmed using standard bisulfite PCR and sequencing. 
errations associated with imprinting disorders and may be relevant to the epigenetic causes of common diseases.

\section{Methods}

\section{Tissue samples and cell lines}

Peripheral blood was obtained from healthy volunteers or from the umbilical cord of newborns for which we obtained matched placental biopsies. These samples were collected at the Hospital St. Joan De Deu (Barcelona, Spain) and the National Center for Child Health and Development (Tokyo, Japan). All placenta-derived DNA samples were free of maternal DNA contamination based on microsatellite repeat analysis. The brain samples were obtained from BrainNet Europe/Barcelona Brain Bank. Ethical approval for this study was granted by the Institutional Review Boards at the National Center for Child Health and Development (project 234), Saga University (21-5), Hamamatsu University School of Medicine (23-12), Hospital St. Joan De Deu Ethics Committee (35/07), and Bellvitge Institute for Biomedical Research (PR006/08). Written informed consent was obtained from all participants.

The hES (SHEF 3, 5, 6 and Val10B) and parthenogenetically activated oocyte (LLC6P, LLC7P, LLC8P, and LLC9P) cell lines were used because they were epigenetically stable at imprinted loci (with the exception of NNAT LOM and GNAS GOM in LLC7P; LOM of PEG3 in Val10B; GOM of MCTS2P in SHEF3) and grown as previously described (Harness et al. 2011). Ethical approval for the study of these cells was granted by the Bellvitge Institute for Biomedical Research Ethics Committee (PR096/10) and Comité Ético de Investigación Clínica (CEIC) del Centro de Medicina Regenerativa de Barcelona-CMR[B] (28/2012) and complied with the legal guidelines outlined by the Generalitat de Catalunya El conseller de Slaut.

Wild-type mouse embryos and placentae were produced by crossing C57BL/6 (B) with Mus musculus molosinus (JF1) or Mus musculus castaneous (C) mice. Mouse work was approved by the Institutional Review Board Committees at the National Center for Child Health and Development (approval number A2010-002). Animal husbandry and breeding were conducted according to the institutional guidelines for the care and the use of laboratory animals. DNA and RNA extractions and cDNA synthesis were carried out as previously described (Monk et al. 2006).

\section{Characterization of the genome-wide UPD samples}

Genomic DNA was isolated from two previously described genome-wide paternal UPD cases with BWS features (Romanelli et al. 2011) and two newly identified individuals, at Saga University, as well as one genome-wide maternal UPD with a SRS phenotype (Yamazawa et al. 2010). Each of these cases had undergone extensive molecular characterization to confirm genome-wide UPD status and the extent of mosaicism. We used DNA isolated from lymphocytes, as these samples had minimal contamination of the biparental cell lines. The genome-wide pUPD samples had $9,11,9$, and $2 \%$ biparental contribution, whereas the genomewide SRS sample had $16 \%$. In addition, four hydatidiform moles were collected by the National Center for Child Health and Development.

\section{Genome-wide methylation profiling}

We analyzed six publicly available methylomes, including those derived from CD4+ lymphocytes (GSE31263) (Heyn et al. 2012), brain (GSM913595) (Zeng et al. 2012), the H1 hES cell line (GSM432685, GSM432686, GSM429321, GSM429322, GSM429323), and sperm
(GSE30340). In addition, we generated three additional tissue methylomes using WGBS for brain, liver, and placenta. WGBS libraries were generated as previously described (Heyn et al. 2012).

We also generated methylation data sets using the Illumina Infinium HumanMethylation450 BeadChip arrays, which simultaneously quantifies $\sim 2 \%$ of all CpG dinucleotides. Bisulfite conversion of $600 \mathrm{ng}$ of DNA was performed according to the manufacturer's recommendations for the Illumina Infinium Assay (EZ DNA methylation kit, Zymo). The bisulfite-converted DNA was used for hybridization following the Illumina Infinium HD methylation protocol at genomic facilities of the Cancer Epigenetics and Biology Program (Barcelona, Spain) or the National Center for Child Health and Development. Data was generated for the genome-wide UPDs $(4 \times$ pUPD, $1 \times$ mUPD), two brain, one liver, one muscle, one pancreas, two sperm, four hydatidiform moles, four term placentae, four phES cell lines, and the four hES lines. In addition, we used three leukocyte data sets from GSE30870.

\section{Data filtering and analysis}

For WGBS, the sequence reads were aligned to either strand of the hg19 reference genome using a custom computational pipeline (autosomal CpGs with at least five reads: brain sample, 190,314,071 aligned unique reads, 83\% coverage; liver sample, $778,733,789$ aligned unique reads, $96.6 \%$ coverage; placenta sample, 319,362,653 aligned unique reads, $89.6 \%$ coverage). The methylation level of each cytosine within CpG dinucleotides was estimated as the number of reads reporting a $C$, divided by the total number of reads reporting a $\mathrm{C}$ or $\mathrm{T}$. For the identification of intermediately methylated regions associated with imprinted DMRs, we performed a sliding window approach in which the methylation of $25 \mathrm{CpGs}$ was averaged after filtering for repetitive sequences. The location of these sequences was taken from the UCSC sequence browser. An interval was considered partially methylated if the average methylation was $0.25<$ mean $\pm 1.5 \mathrm{SD}<0.75$.

For the Illumina Infinium HumanMethylation 450 BeadChip array, before analyzing the data, we excluded possible sources of technical biases that could influence results. We applied signal background subtraction, and inter-plate variation was normalized using default control probes in BeadStudio (version 2011.1_Infinium HD). We discarded probes with a detection $P$-value $>0.01$. We also excluded probes that lacked signal values in one or more of the DNA samples analyzed. In addition, we discarded 16,631 probes as they contained SNPs present in $>1 \%$ of the population (dbSNP 137). Lastly, prior to screening for novel imprinted DMRs, we excluded all X chromosome CpG sites. In total, we analyzed 442,772 probes in all DNA samples. All hierarchical clustering and $\beta$-value evaluation was performed using the Cluster Analysis tool of the BeadStudio software.

In-house R-package scripts were used to evaluate the average methylation of three contiguous Infinium probes. To identify regions with potential allelic methylation, we screened the reciprocal genome-wide UPDs for three consecutive probes with an average $\beta$-value difference greater than 0.3 (Limma linear model $P<0.05)$ :

$$
\left|\frac{1}{3} \sum_{n=0}^{2} p U P D s_{n}-\frac{1}{3} \sum_{n=0}^{2} m U P D_{n}\right|>0.3 .
$$

With the condition that the average of three consecutive probes for the normal leukocytes is between the values for the reciprocal genome-wide UPDs:

\section{Genome Research}




$$
\left\{\begin{array}{l}
\text { if } \frac{1}{3} \sum_{n=0}^{2} p U P D s_{n}>\frac{1}{3} \sum_{n=0}^{2} m U P D_{n} \\
\text { then } \frac{1}{3} \sum_{n=0}^{2} p U P D s_{n}>\frac{1}{3} \sum_{n=0}^{2} \text { Leukocytes }_{n}>\frac{1}{3} \sum_{n=0}^{2} \text { mUPD }_{n} \\
\text { if } \frac{1}{3} \sum_{n=0}^{2} m U P D_{n}>\frac{1}{3} \sum_{n=0}^{2} p U P D s_{n} \\
\text { then } \frac{1}{3} \sum_{n=0}^{2} \text { mUPD }_{n}>\frac{1}{3} \sum_{n=0}^{2} \text { Leukocytes }_{n}>\frac{1}{3} \sum_{n=0}^{2} \text { pUPD }_{n} .
\end{array}\right.
$$

The final condition was that the average of three consecutive probes for normal leukocytes is within the $0.25-0.75$ intermediate methylation range:

$$
0.25>\frac{1}{3} \sum_{n=0}^{2} \text { Leukocytes }_{n}>0.75
$$

\section{Genotyping and imprinting analysis}

Genotypes of potential SNPs identified in the UCSC Genome Browser (hg19) were obtained by PCR and direct sequencing. Sequence traces were interrogated using Sequencher v4.6 (Gene Codes Corporation) to distinguish heterozygous and homozygous samples. Heterozygous sample sets were analyzed for either allelic expression using RT-PCR or bisulfite PCR, incorporating the polymorphism within the final PCR amplicon so that parental alleles could be distinguished (for primer sequence, see Supplemental Table S3).

\section{Bisulfite PCR}

Approximately $1 \mu \mathrm{g}$ DNA was subjected to sodium bisulfite treatment and purified using the EZ DNA Methylation-Gold kit (Zymo), and was used for all bisulfite PCR analysis. Approximately $2 \mu \mathrm{L}$ of bisulfite-converted DNA was used in each amplification reaction using Immolase Taq polymerase (Bioline) at 35-45 cycles, and the resulting PCR product cloned into pGEM-T easy vector (Promega) for subsequent subcloning and sequencing (for primer sequence, see Supplemental Table S3). For the confirmation of an imprinted DMR, we analyzed a minimum of three heterozygous samples and, where possible, two different tissues.

\section{Chromatin immunoprecipitation (ChIP)}

We analyzed publicly available H3K4me3 ChIP-seq and meDIP-seq data sets, including those derived from lymphocytes (GSM772948, GSM772836, GSM772916, GSM543025, GSM613913), brain (GSM806943, GSM806935, GSM806948, GSM669614, GSM669615), and the H1 hES cell line (GSM409308, GSM469971, GSM605315, GSM428289, GSM456941, GSM543016). For H3K9me3 in hES cells, we used GSM450266. In addition, we used the sperm ChIP-seq data set for H3K4me3 as a direct measure of nucleosome occupancy (GSM392696, GSM392697, GSM392698, GSM392714, GSM392715, GSM392716) (Hammoud et al. 2009).

The confirmation of allelic H3K4me3 in leukocytes or lymphoblastoid cell lines was performed as previously described (Iglesias-Platas et al. 2013). Briefly, $100 \mu \mathrm{g}$ of chromatin was used for an immunoprecipitation reaction with Protein A agarose/ salmon sperm DNA (16-157, Millipore) and a H3K4me3 (07-473, Millipore). Each ChIP was performed in triplicate alongside a mock immunoprecipitation with an unrelated IgG antiserum, and a $1 \%$ fraction of the input chromatin was extracted in parallel. Levels of immunoprecipitated chromatin at each specific region were determined by qPCR using SYBR Green (Applied Biosystems) carried out on the Applied Biosystems 7900 Fast real-time
PCR system (for primer sequence, see Supplemental Table S3). Each PCR was run in triplicate and protein binding was quantified as a percentage of total input material.

\section{Data access}

The data from this study have been submitted to the NCBI Gene Expression Omnibus (GEO; http://www.ncbi.nlm.nih.gov/geo/) under accession number GSE52578.

\section{List of affiliations}

${ }^{1}$ Imprinting and Cancer Group, Cancer Epigenetic and Biology Program, Institut d'Investigació Biomedica de Bellvitge, Hospital Duran i Reynals, 08908 Barcelona, Spain; ${ }^{2}$ Department of Maternal-Fetal Biology, National Research Institute for Child Health and Development, Tokyo 157-8535, Japan; ${ }^{3}$ Servicio de Neonatología, Hospital Sant Joan de Déu, Fundació Sant Joan de Déu, 08950 Barcelona, Spain; ${ }^{4}$ Department of Systems Biomedicine, National Research Institute for Child Health and Development, Tokyo 157-8535, Japan; ${ }^{5}$ Fundación IVI-Instituto Universitario IVI-Universidad de Valencia, INCLIVA, 46980 Paterna, Valencia, Spain; ${ }^{6}$ Centre for Stem Cell Biology, Department of Biomedical Science, University of Sheffield, Sheffield S10 2TN, United Kingdom; ${ }^{7}$ Reeve-Irvine Research Centre, Sue and Bill Gross Stem Cell Research Center, Department of Anatomy and Neurobiology, School of Medicine, University of California at Irvine, Irvine, California 92697, USA; ${ }^{8}$ Cancer Epigenetics Group, Cancer Epigenetic and Biology Program, Institut d'Investigació Biomedica de Bellvitge, Hospital Duran i Reynals, 08908 Barcelona, Spain; ${ }^{9}$ Department of Obstetrics and Gynecology, Graduate School of Medical Science, Kyushu University, Fukuoka 812-8582, Japan; ${ }^{10}$ Instituto de Genética Médica y Molecular, CIBERER, IDIPAZ-Hospital Universitario La Paz, Universidad Autónoma de Madrid, 28046 Madrid, Spain; ${ }^{11}$ Division of Molecular Genetics and Epigenetics, Department of Biomolecular Sciences, Faculty of Medicine, Saga University, Saga 849-8501, Japan; ${ }^{12}$ Department of Physiological Sciences II, School of Medicine, University of Barcelona, 08036 Barcelona, Catalonia, Spain; ${ }^{13}$ Institucio Catalana de Recerca i Estudis Avançats (ICREA), 08010 Barcelona, Catalonia, Spain; ${ }^{14}$ Department of Pediatrics, Hamamatsu University School of Medicine, Hamamatsu 431-3192, Japan.

\section{Acknowledgments}

We thank S. Morán at PEBC-IDIBELL for performing the methylation array hybridization and S. Sayols and H. Heyn for bioinformatics assistance. We also thank P. Arnaud and J. Frost for stimulating discussions and helpful comments. This work was partially funded by grants from the Fundació La Marató de TV3 (grant no. 101130 to D.M. and P.L.); Spanish Ministerio de Educacion y Ciencia (BFU2011-27658 to D.M.); JST/CREST and the Health and Labour Sciences Research Grant (Nanbyo-Ippan-003 to K.H.); the Ministry of Education, Culture, Sports, Science, and Technology of Japan (\#22249010) and the National Center for Child Health and Development (\#24-3) to K.N. D.M. is a Ramon y Cajal research fellow. Finally, we would like to thank all the patients and their families for participating in this project.

\section{References}

Amor DJ, Halliday J. 2008. A review of known imprinting syndromes and their association with assisted reproduction technologies. Hum Reprod 23: 2826-2834. 
Baglivo I, Esposito S, De Cesare L, Sparago A, Anvar Z, Riso V, Cammisa M, Fattorusso R, Grimaldi G, Riccio A, et al. 2013. Genetic and epigenetic mutations affect the DNA binding capability of human ZFP57 in transient neonatal diabetes type 1. FEBS Lett 587: 1474-1481.

Barbaux S, Gascoin-Lachambre G, Buffat C, Monnier P, Mondon F, Tonanny MB, Pinard A, Auer J, Bessières B, Barlier A, et al. 2012. A genome-wide approach reveals novel imprinted genes expressed in the human placenta. Epigenetics 7: 1079-1090.

Bourc'his D, Xu GL, Lin CS, Bollman B, Bestor TH. 2001. Dnmt3L and the establishment of maternal genomic imprints. Science 294: 2536-2539.

Buiting K. 2010. Prader-Willi syndrome and Angelman syndrome. Am J Med Genet C Semin Med Genet 154C: 365-376.

Choufani S, Shuman C, Weksberg R. 2010. Beckwith-Wiedemann syndrome. Am J Med Genet C Semin Med Genet 154C: 343-354.

Ciccone DN, Su H, Hevi S, Gay F, Lei H, Bajko J, Xu G, Li E, Chen T. 2009 KDM1B is a histone H3K4 demethylase required to establish maternal genomic imprints. Nature 461: 415-418.

Constância M, Kelsey G, Reik W. 2004. Resourceful imprinting. Nature 432: 53-57.

Coombes C, Arnaud P, Gordon E, Dean W, Coar EA, Williamson CM, Feil R, Peters J, Kelsey G. 2003. Epigenetic properties and identification of an imprint mark in the Nesp-Gnasxl domain of the mouse Gnas imprinted locus. Mol Cell Biol 23: 5475-5488.

Cooper WN, Constância M. 2010. How genome-wide approaches can be used to unravel the remaining secrets of the imprintome. Brief Funct Genomics 9: 315-328.

Das R, Lee YK, Strogantsev R, Jin S, Lim YC, Ng PY, Lin XM, Chng K, Yeo GSh, Ferguson-Smith AC, et al. 2013. DNMT1 and AIM1 imprinting in human placenta revealed through a genome-wide screen for allelespecific DNA methylation. BMC Genomics 14: 685.

Dhayalan A, Rajavelu A, Rathert P, Tamas R, Jurkowska RZ, Ragozin S, Jeltsch A. 2010. The Dnmt3a PWWP domain reads histone 3 lysine 36 trimethylation and guides DNA methylation. J Biol Chem 285: 2611426120.

Eggermann T. 2010. Russell-Silver syndrome. Am J Med Genet C Semin Med Genet 154C: $355-364$

Ehrlich M, Gama-Sosa MA, Huang LH, Midgett RM, Kuo KC, McCune RA, Gehrke C. 1982. Amount and distribution of 5-methylcytosine in human DNA from different types of tissues of cells. Nucleic Acids Res 10: 2709-2721.

El-Maarri O, Buiting K, Peery EG, Kroisel PM, Balaban B, Wagner K, Urman B, Heyd J, Lich C, Brannan CI, et al. 2001. Maternal methylation imprints on human chromosome 15 are established during or after fertilization. Nat Genet 27: 41-44.

Fuke C, Shimabukuro M, Petronis A, Sugimoto J, Oda T, Miura K, Miyazaki T, Ogura C, Okazaki Y, Jinno Y. 2004. Age related changes in 5-methylcytosine content in human peripheral leukocytes and placentas: An HPLC-based study. Ann Hum Genet 68: 196-204.

Geuns E, De Temmerman N, Hilven P, Van Steirteghem A, Liebaers I, De Rycke M. 2007. Methylation analysis of the intergenic differentially methylated region of DLK1-GTL2 in human. Eur J Hum Genet 15: 352 361 .

Gregg C, Zhang J, Weissbourd B, Luo S, Schroth GP, Haig D, Dulac C. 2010. High-resolution analysis of parent-of-origin allelic expression in the mouse brain. Science 329: 643-648.

Hammoud SS, Nix DA, Zhang H, Purwar J, Carrell DT, Cairns BR. 2009 Distinctive chromatin in human sperm packages genes for embryo development. Nature 460: 473-478.

Harness JV, Turovets NA, Seiler MJ, Nistor G, Altun G, Agapova LS, Ferguson D, Laurent LC, Loring JF, Keirstead HS. 2011. Equivalence of conventionallyderived and parthenote-derived human embryonic stem cells. PLoS ONE 6: e14499.

Hata K, Okano M, Lei H, Li E. 2002. Dnmt3L cooperates with the Dnmt3 family of de novo DNA methyltransferases to establish maternal imprints in mice. Development 129: 1983-1993.

Hayashizaki Y, Shibata H, Hirotsune S, Sugino H, Okazaki Y, Sasaki N, Hirose K, Imoto H, Okuizumi H, Muramatsu M, et al. 1994. Identification of an imprinted U2af binding protein related sequence on mouse chromosome 11 using the RLGS method. Nat Genet 6: 33-40.

Heyn H, Li N, Ferreira HJ, Moran S, Pisano DG, Gomez A, Diez J, SanchezMut JV, Setien F, Carmona FJ, et al. 2012. Distinct DNA methylomes of newborns and centenarians. Proc Natl Acad Sci 109: 10522-10527.

Hiura H, Sugawara A, Ogawa H, John RM, Miyauchi N, Miyanari Y, Horiike T, Li Y, Yaegashi N, Sasaki H, et al. 2010. A tripartite paternally methylated region within the Gpr1-Zdbf2 imprinted domain on mouse chromosome 1 identified by meDIP-on-chip. Nucleic Acids Res 38: 4929-4945.

Kanber D, Berulava T, Ammerpohl O, Mitter D, Richter J, Siebert R, Horsthemke B, Lohman D, Buiting K. 2009. The human retinoblastoma gene is imprinted. PLoS Genet 12: e1000790.

Iglesias-Platas I, Court F, Camprubi C, Sparago A, Guillaumet-Adkins A, Martin-Trujillo A, Riccio A, Moore GE, Monk D. 2013. Imprinting at the
PLAGL1 domain is contained within a 70-kb CTCF/cohesin-mediated non-allelic chromatin loop. Nucleic Acids Res 41: 2171-2179.

Kagami M, Sekita Y, Nishimura G, Irie M, Kato F, Okada M, Yamamori S, Kishimoto H, Nakayama M, Tanaka Y, et al. 2008. Deletions and epimutations affecting the human $14 \mathrm{q} 32.2$ imprinted region in individuals with paternal and maternal upd(14)-like phenotypes. Nat Genet 40: $237-242$.

Kagami M, O'Sullivan MJ, Green AJ, Watabe Y, Arisaka O, Masawa N, Matsuoka K, Fukami M, Matsubara K, Kato F, et al. 2010. The IG-DMR and the MEG3-DMR at human chromosome 14q32.2: Hierarchical interaction and distinct functional properties as imprinting control centers. PLoS Genet 6: e1000992.

Kelsey G. 2010. Imprinting on chromosome 20: Tissue-specific imprinting and imprinting mutations in the GNAS locus. Am J Med Genet C Semin Med Genet 154C: 377-386.

Kelsey G, Bodle D, Miller HJ, Beechey CV, Coombes C, Peters J, Williamson CM. 1999. Identification of imprinted loci by methylation-sensitive representational difference analysis: Application to mouse distal chromosome 2. Genomics 62: 129-138.

Kobayashi H, Sakurai T, Imai M, Takahashi N, Fukuda A, Yayoi O, Sato S, Nakabayashi K, Hata K, Sotomaru Y, et al. 2012. Contribution of intragenic DNA methylation in mouse gametic DNA methylomes to establish oocyte-specific heritable marks. PLoS Genet 8: e1002440.

Kobayashi H, Yanagisawa E, Sakashita A, Sugawara N, Kumakura S, Ogawa H, Akutsu H, Hata K, Nakabayashi K, Kono T. 2013. Epigenetic and transcriptional features of the novel human imprinted lncRNA GPR1AS suggest it is a functional ortholog to mouse Zdbf2linc. Epigenetics 8: 635645.

Kong A, Steinthorsdottir V, Masson G, Thorleifsson G, Sulem P, Besenbacher S, Jonasdottir A, Sigurdsson A, Kristinsson KT, Jonasdottir A, et al. 2009. Parental origin of sequence variants associated with complex diseases. Nature 462: 868-874.

Lapunzina P, Monk D. 2011. The consequences of uniparental disomy and copy number neutral loss-of-heterozygosity during human development and cancer. Biol Cell 103: 303-317.

Li X, Ito M, Zhou F, Youngson N, Zuo X, Leder P, Ferguson-Smith AC. 2008. A maternal-zygotic effect gene, $Z f p 57$, maintains both maternal and paternal imprints. Dev Cell 15: 547-557.

Lister R, Pelizzola M, Dowen RH, Hawkins RD, Hon G, Tonti-Filippini J, Nery JR, Lee L, Ye Z, Ngo QM, et al. 2009. Human DNA methylomes at base resolution show widespread epigenomic differences. Nature 462: 315322.

Lopes S, Lewis A, Hajkova P, Dean W, Oswald J, Forné T, Murrell A, Constância M, Bartolomei M, Walter J, et al. 2003. Epigenetic modifications in an imprinting cluster are controlled by a hierarchy of DMRs suggesting long-range chromatin interactions. Hum Mol Genet 12: 295-305.

Mackay DJ, Temple IK. 2010. Transient neonatal diabetes mellitus type 1. Am J Med Genet C Semin Med Genet 154C: 335-342.

Mai Q, Yu Y, Li T, Wang L, Chen MJ, Huang SZ, Zhou C, Zhou Q. 2007. Derivation of human embryonic stem cell lines from parthenogenetic blastocysts. Cell Res 17: 1008-1012.

Molaro A, Hodges E, Fang F, Song Q, McCombie WR, Hannon GJ, Smith AD. 2011. Sperm methylation profiles reveal features of epigenetic inheritance and evolution in primates. Cell 146: 1029-1041.

Monk D. 2010. Deciphering the cancer imprintome. Brief Funct Genomics 9: 329-339.

Monk D, Arnaud P, Apostolidou S, Hills FA, Kelsey G, Stanier P, Feil R, Moore GE. 2006. Limited evolutionary conservation of imprinting in the human placenta. Proc Natl Acad Sci 103: 6623-6628.

Nakabayashi K, Trujillo AM, Tayama C, Camprubi C, Yoshida W, Lapunzina P, Sanchez A, Soejima H, Aburatani H, Nagae G, et al. 2011. Methylation screening of reciprocal genome-wide UPDs identifies novel humanspecific imprinted genes. Hum Mol Genet 20: 3188-3197.

Nakamura T, Arai Y, Umehara H, Masuhara M, Kimura T, Taniguchi H, Sekimoto T, Ikawa M, Yoneda Y, Okabe M. 2007. PGC7/Stella protects against DNA demethylation in early embryogenesis. Nat Cell Biol 9: 6471.

Noguer-Dance M, Abu-Amero S, Al-Khtib M, Lefèvre A, Coullin P, Moore GE, Cavaillé J. 2010. The primate-specific microRNA gene cluster (C19MC) is imprinted in the placenta. Hum Mol Genet 19: 3566-3582.

Okae H, Hiura H, Nishida Y, Funayama R, Tanaka S, Chiba H, Yaegashi N, Nakayama K, Sasaki H, Arima T. 2011. Re-investigation and RNA sequencing-based identification of genes with placenta-specific imprinted expression. Hum Mol Genet 21: 548-558.

Park KY, Sellars EA, Grinberg A, Huang SP, Pfeifer K. 2004. The H19 differentially methylated region marks the parental origin of a heterologous locus without gametic DNA methylation. Mol Cell Biol 24: 3588-3595.

Proudhon C, Duffié R, Ajjan S, Cowley M, Iranzo J, Carbajosa G, Saadeh H, Holland ML, Oakey RJ, Rakyan VK, et al. 2012. Protection against de

\section{Genome Research}


novo methylation is instrumental in maintaining parent-of-origin methylation inherited from the gametes. Mol Cell 47: 909-920.

Quenneville S, Verde G, Corsinotti A, Kapopoulou A, Jakobsson J, Offner S, Baglivo I, Pedone PV, Grimaldi G, Riccio A, et al. 2011. In embryonic stem cells, ZFP57/KAP1 recognize a methylated hexanucleotide to affect chromatin and DNA methylation of imprinting control regions. Mol Cell 44: $361-372$.

Ramowitz LK, Bartolomei MS. 2011. Genomic imprinting: Recognition and marking of imprinted loci. Curr Opin Genet Dev 22: 72-78.

Romanelli V, Nevado J, Fraga M, Trujillo AM, Mori MÁ, Fernández L, Pérez de Nanclares G, Martínez-Glez V, Pita G, Meneses H, et al. 2011. Constitutional mosaic genome-wide uniparental disomy due to diploidisation: An unusual cancer-predisposing mechanism. J Med Genet 48: 212-216.

Schroeder DI, Blair JD, Lott P, Yu HO, Hong D, Crary F, Ashwood P, Walker C, Korf I, Robinson WP, et al. 2013. The human placenta methylome. Proc Natl Acad Sci 110: 6037-6042.

Sharp A, Migliavacca E, Dupre Y, Stathaki E, Reza Sailani M, Baumer A, Schinzel A, Mackay DJ, Robinson DO, Cobellis G, et al. 2010. Methylation profiling in individuals with uniparental disomy identify novel differentially methylated regions on chromosome 15. Genome Res 20: $1271-1278$.

Smallwood SA, Tomizawa S, Krueger F, Ruf N, Carli N, Segonds-Pichon A, Sato S, Hata K, Andrews SR, Kelsey G. 2011. Dynamic CpG island methylation landscape in oocytes and pre-implantation embryos. Nat Genet 43: 811-814.

Smith ZD, Chan MM, Mikkelsen TS, Gu H, Gnirke A, Regev A, Meissner A. 2012. A unique regulatory phase of DNA methylation in the early mammalian embryo. Nature 484: 339-344.

Thomson JP, Skene PJ, Selfridge J, Clouaire T, Guy J, Webb S, Kerr AR, Deaton A, Andrews R, James KD, et al. 2010. CpG islands influence chromatin structure via the CpG-binding protein Cfp1. Nature 464: 1082-1086.
Tomizawa S, Kobayashi H, Watanabe T, Andrews S, Hata K, Kelsey G, Sasaki H. 2011. Dynamic stage-specific changes in imprinted differentially methylated regions during early mammalian development and prevalence of non-CpG methylation in oocytes. Development 138: 811-820.

Umlauf D, Goto Y, Cao R, Cerqueira F, Wagschal A, Zhang Y, Feil R. 2004. Imprinting along the Kcnq1 domain on mouse chromosome 7 involves repressive histone methylation and recruitment of Polycomb group complexes. Nat Genet 36: 1296-1300.

Wood AJ, Schulz R, Woodfine K, Koltowska K, Beechey CV, Peters J, Bourc'his D, Oakey RJ. 2008. Regulation of alternative polyadenylation by genomic imprinting. Genes Dev 22: 1141-1146.

Xie W, Barr CL, Kim A, Yue F, Lee AY, Eubanks J, Dempster EL, Ren B. 2012. Base-resolution analyses of sequence and parent-of-origin dependent DNA methylation in the mouse genome. Cell 148: 816-831.

Yamazawa K, Nakabayashi K, Kagami M, Sato T, Saitoh S, Horikawa R, Hizuka N, Ogata T. 2010. Parthenogenetic chimaerism/mosaicism with a Silver-Russell syndrome-like phenotype. J Med Genet 47: 782-785.

Yuen RKC, Jiang R, Penaherrera M, McFadeen DE, Robinson WP. 2011. Genome-wide mapping of imprinted differentially methylated regions by DNA methylation profiling of human placentas from triploidies. Epigenetics \& Chromatin 4: 10.

Zeng J, Konopka G, Hunt BG, Preuss TM, Geschwind D, Yi SV. 2012. Divergent whole-genome methylation maps of human and chimpanzee brains reveal epigenetic basis of human regulatory evolution. Am J Hum Genet 91: 455-465.

Zhang Y, Jurkowska R, Soeroes S, Rajavelu A, Dhayalan A, Bock I, Rathert P, Brandt O, Reinhardt R, Fischle W, et al. 2010. Chromatin methylation activity of Dnmt3a and Dnmt3a/3L is guided by interaction of the ADD domain with the histone H3 tail. Nucleic Acids Res 38: 4246-4253.

Received August 9, 2013; accepted in revised form December 26, 2013. 


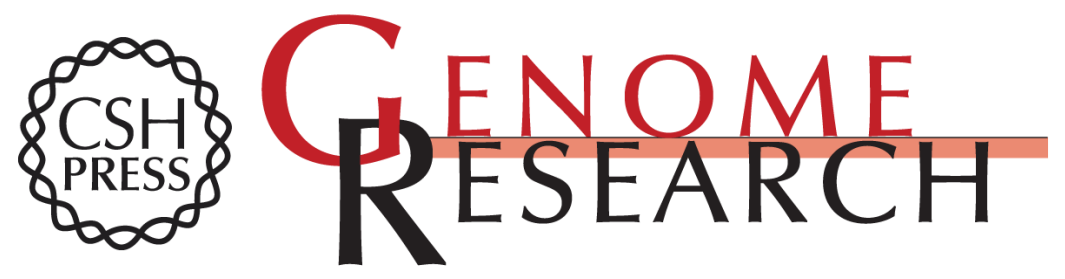

\section{Genome-wide parent-of-origin DNA methylation analysis reveals the intricacies of human imprinting and suggests a germline methylation-independent mechanism of establishment}

Franck Court, Chiharu Tayama, Valeria Romanelli, et al.

Genome Res. 2014 24: 554-569 originally published online January 8, 2014

Access the most recent version at doi:10.1101/gr.164913.113

Supplemental
Material http://genome.cshlp.org/content/suppl/2014/01/15/gr.164913.113.DC1

References This article cites 64 articles, 13 of which can be accessed free at:

http://genome.cshlp.org/content/24/4/554.full.html\#ref-list-1

Creative This article is distributed exclusively by Cold Spring Harbor Laboratory Press for the Commons first six months after the full-issue publication date (see

License http://genome.cshlp.org/site/misc/terms.xhtml). After six months, it is available under a Creative Commons License (Attribution-NonCommercial 3.0 Unported), as described at http://creativecommons.org/licenses/by-nc/3.0/.

Email Alerting Receive free email alerts when new articles cite this article - sign up in the box at the Service top right corner of the article or click here.

\section{Affordable, Accurate Sequencing.}

To subscribe to Genome Research go to:

https://genome.cshlp.org/subscriptions 